\title{
OBSERVATIONS \\ SUR LES QUALITÉS ORGANOLEPTIQUES DU LAIT
}

par

\section{S. KUZDZAL-SAVOIE et G. MOCQUOT}

Station centrale de Recherches laitières et de Technologie des produits animaux Jouy-en-Josas

\section{DeUXIÈme PARTIE}

\section{TRAVAUX PERSONNELS}

Il nous a semblé utile de reprendre en France une étude sur les défauts de goût susceptibles d'apparaître dans le lait. Retrouverait-on les mêmes défauts que ceux signalés par les auteurs étrangers? Serait-il possible de mettre en évidence certains facteurs responsables de l'apparition de ces défauts ?

Les résultats obtenus au cours de l'étude poursuivie de janvier 1955 à mars 1957, au Centre National de Recherches Zootechniques de Jouy-en-Josas, permettent de répondre partiellement à ces questions, et nous allons les exposer et les discuter maintenant.

\section{A. - Méthode expérimentale}

De janvier 1955 à mars 1957, nous avons procédé chaque semaine à des examens organoleptiques sur des laits individuels, provenant de vaches du troupeau du C.N.R.Z. de Jouy-en-Josas,

Il y a lieu de distinguer deux périodes : l'une de fin janvier 1955 à fin janvier 1956, l'autre de février 1956 à mars 1957.

Au cours de ces deux périodes, les modalités du prélèvement des échantillons et celles de l'examen organoleptique ont été légèrement différentes.

\section{I. - Prélièvement}

Les échantillons provenaient de laits individuels, prélevés en moyenne une fois par semaine. En 1955, les prélèvements avaient lieu lors de la traite du matin et lors de la traite du soir. En 1956, ils étaient effectués, d'une façon générale, lors de la traite du soir.

En 1955, on a examiné, avec une fréquence variable, des laits 
de toutes les vaches en lactation, c'est-à-dire : 5 vaches de race Pie Rouge de l'Est, 22 vaches de race Normande et 24 vaches de race Frisonne Pie Noire.

Au cours de la deuxième période d'observation (1956-1957), les laits de 15 vaches (8 Frisonne Pie Noire, 6 Normandes et 1 Pie Rouge de l'Est) ont été examinés chaque semaine. Par contre, l'examen organoleptique des laits de toutes les vaches en lactation a été effectué quatre fois seulement : en mars 1956, en janvier, février et mars 1957.

Pour chaque vache, on prélevait, dans deux bouteilles en verre, un échantillon d'un litre et un échantillon d'un demi-litre sur la totalité du lait de la traite.

Aussitôt après la traite, les échantillons de lait étaient rassemblés, les bouteilles capsulées et placées à la glacière ( 2 à $\left.4^{\circ} \mathrm{C}\right)$.

Une pasteurisation basse, au bain-marie (30 minutes à $63^{\circ} \mathrm{C}$ ), était effectuée sur les bouteilles contenant un litre de lait, à 4 5 heures environ après la traite du matin et 16 à 17 heures après la traite du soir. Les échantillons pasteurisés et refroidis étaient ensuite placés à la glacière.

L'examen organoleptique avait lieu le surlendemain, soit 48 heures après la pasteurisation et concernait le lait cru d'une part, le lait pasteurisé d'autre part.

\section{2. - Examen organoleptique}

Les échantillons ont été examinés en 1955, par un seul expert et en 1956, par un groupe de 4 à 6 experts ; les appréciations personnelles étaient confrontées, et au besoin, révisées, avant la rédaction de l'appréciation finale.

L'examen organoleptique était pratiqué sur les échantillons de lait réchauffés à $30-35^{\circ} \mathrm{C}$.

$\mathrm{Au}$ cours de l'examen organoleptique, nous avons relevé les défauts de goût bien connus : goût de rance, saveur oxydée (ce terme étant réservé à la saveur métallique, au goût de papier ou de carton), enfin goût de "nourriture». Certains goûts provenant de la nourriture étaient facilement discernables dans certains cas (goût de betterave, d'ensilage, d'herbe) et dans d'autres cas, n'ont pu être attribués avec précision à tel ou tel aliment.

Nous avons rencontré des laits présentant un goût spécial que nous avons parfois défini par analogie (goût d'eau de vaisselle, de bois, de terre, de feuilles mortes, de champignon) et que plus souvent, nous n'avons pas su définir. Le goût de cuit a été assez souvent rencontré ,les goûts de brûlé et de caramel très rarement. Le goût de cuit est délicat à apprécier : quand on examine un grand nombre 
d'échantillons à la fois, on s'y habitue en effet très vite. Dans l'estimation statistique de l'ensemble des résultats de nos observations, seul un goût de cuit très prononcé, et rendant le lait nettement désagréable à boire, a été appelé défaut. Le reste du temps, nous avons noté l'apparition du goût de cuit mais le lait a été considéré comme passable.

Les différents défauts de goût que nous venons d'énumérer peuvent se rencontrer simultanément sur le même échantillon. Ainsi nous avons relevé les associations suivantes : oxydé-nourriture, oxydé-betterave, rance-betterave, betterave-ensilage.

Nous avons de plus rencontré des laits présentant une saveur sucrée ou salée. La saveur salée n'a pas été considérée comme un défaut.

Au cours de l'examen organoleptique, nous avons distingué les "très bons laits" des "bons laits " et des laits "assez bons ". Mais nous avons rassemblé ces trois catégories et qualifié l'ensemble de "bons laits" pour la présentation statistique des observations. Nous avons procédé de la même façon pour les laits jugés fades, plats, " ni bons ni mauvais " et passables, que nous avons également rassemblés. Nous les avons parfois réunis aux «bons laits», et parfois nous les avons présentés séparément. Les indications voulues sont données chaque fois que cela est nécessaire dans la suite du texte. Il est important de signaler que l'examen organoleptique était sévère et que les échantillons qui ne sont pas classés dans la catégorie des "bons laits" possédaient cependant des qualités organoleptiques, qui, dans beaucoup de cas les auraient fait considérer comme acceptables pour le consommateur.

\section{B. - Résultats}

\section{1. - Influence de la saison \\ SUR L'APPARITION DES DÉFAUTS DE GOUT}

Le tableau I et les figures $1 a$ et $1 b$ donnent les résultats de l'ensemble des examens organoleptiques que nous avons effectués sur les échantillons de laits individuels crus et pasteurisés, provenant des vaches du troupeau du Centre National de Recherches Zootechniques, pendant les années 1955, 1956, 1957. 1118 échantillons de lait cru et 1053 échantillons de lait pasteurisé ont été examinés.

Dans le tableau I et sur les figures $1 a$ et $1 b$, les laits répondant aux qualifications suivantes : très bon, bon, assez bon, passable, ni bon ni mauvais, plat, fade, aqueux, ont été groupés dans la catégorie " bons laits ". Les laits présentant un goût de rance léger ou 
accentué ont été groupés dans une même catégorie. Il en a été de même pour la saveur oxydée.

On peut constater qu'à aucune période de l'année, le pourcentage maximum de 100 p. 100 de "bons laits" n'est atteint. Les valeurs les plus élevées sont pour les laits crus de 90,7 p. 100 en juillet et pour les laits pasteurisés de 96,2 p. 100 en août.

Les valeurs les plus faibles sont observées pendant la période de stabulation. Les dates de mise à l'herbe du troupeau ont été : 18 avril 1955, 5 mai 1956 et 16 avril 1957. Les dates de rentrée à l'étable : 21 octobre 1955 et période du 22 au 30 octobre 1956. D'octobre à mai, les proportions moyennes de "bons laits" sont de 49,8 p. 100 pour les laits crus et de 63,1 p. 100 pour les laits pasteurisés, alors que ces proportions s'élèvent à 78,7 p. 100 et 82,8 p. 100 pendant la période de mai à octobre.

Goût de rance. - Ce défaut apparaît au cours de toute l'année, mais alors qu'il est noté pendant les mois de mai, juin et juillet sur $4,9,10,5$ et 1,9 p. 100 des échantillons de lait oru examinés, il est relevé pendant les autres mois sur 19,2 à 45,7 p. 100 des échantillons examinés.

Saveur oxydée. - Ce défaut n'est relevé de façon appréciable que de novembre à fin mars. Il est particulièrement accentué en janvier et février.

Goût de nourriture. - Ce défaut se rencontre toute l'année; il paraît cependant plus accentué au printemps et à l'automne. Le défaut de goût d'ensilage s'observe dès novembre, c'est-à-dire dès la distribution de l'ensilage aux vaches laitières.

Goût de cuit. - Ce défaut propre au lait pasteurisé a été relevé souvent au printemps et au début de l'automne.

Les laits présentant un goût spécial où les laits "mauvais» se rencontrent à toute époque de l'année. On note cependant une nette diminution de ces défauts, en juillet et août et une légère accentuation au printemps et à l'automne.

\section{2. - VARIATION DANS L'APPARITION DES DÉfAutS DE GOUT, D'UNE ANNÉE A L'AUTRE}

Si les mêmes défauts de goût apparaissent à peu de chose près aux mêmes périodes, ils peuvent être plus fréquents une année, et moins fréquents une autre année.

Si l'on sépare les deux périodes d'observation (janvier 1955janvier 1956, février 1956-mars 1957), on constate, par exemple, que le goût de betterave qui apparaissait en 1955 sur 21,4 p. 100 
PROPORTIONS DES " BONS LAITS \# ET DES LAITS PRÉSENTANT UN DÉFAUT DE GOUT EXPRIMÉES EN P. GENT DU TOTAL DES EGHANTILLONS EXAMINES

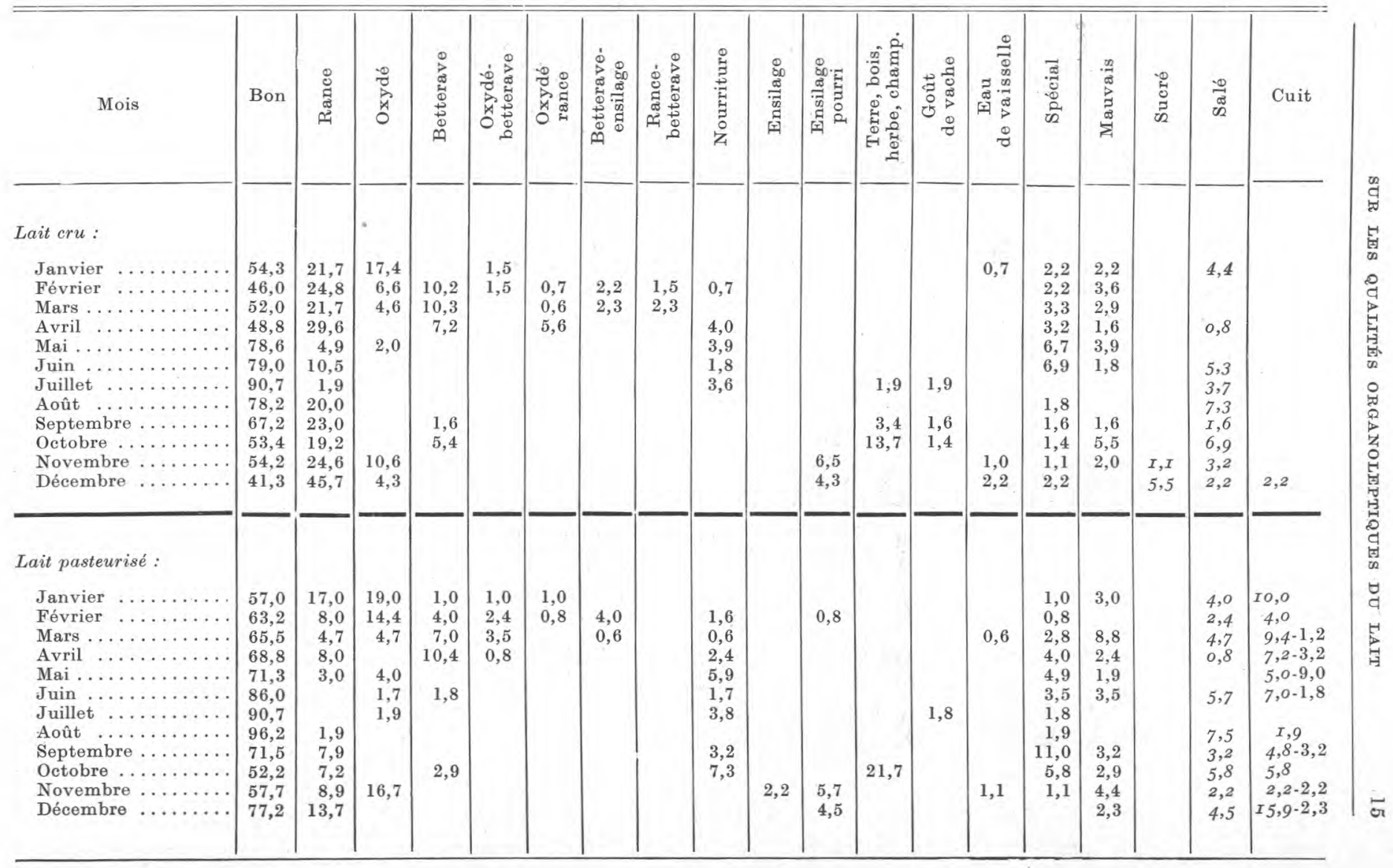

Les échantillons correspondant aux proportions portées en italique ont été classés dans la catégorie "bons laits " (cf. texte, $2^{e}$ partie, A2-examen organoleptique). 


\section{LAITS CRUS}

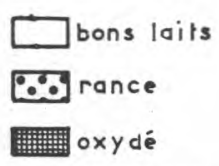

\%ou gout de betherave oxydé rance

oxydé bétt.

rance betl.

betr. ensilage

ensilage pourri

terre

bois herbe

champignons

gout de vache gout deas sale

gour de nourriture goul special

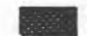

$\%$

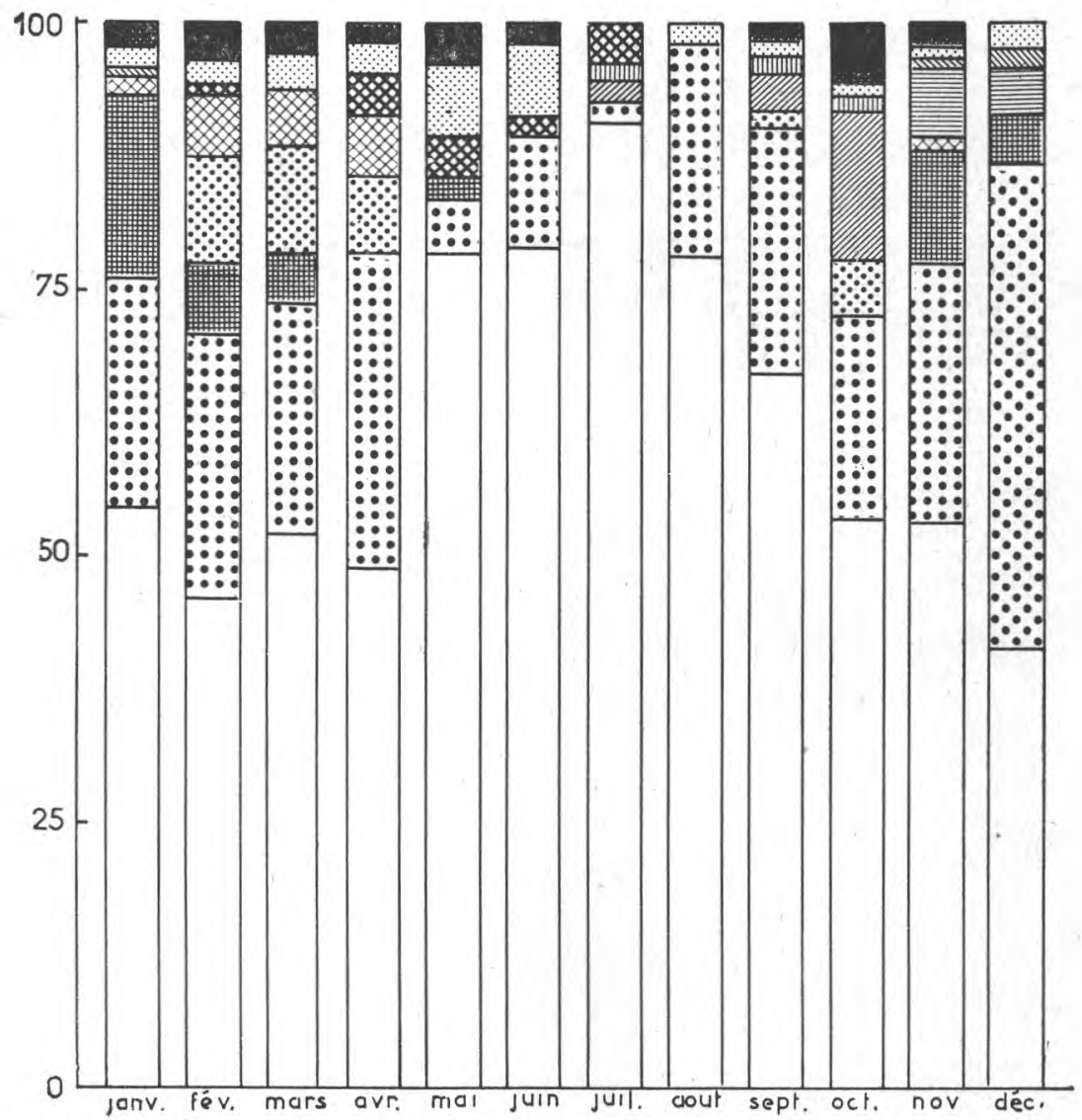

Fig. la. - Proportions de "bons laits " et de laits présentant un défaut de goût, exprimées en pourcentage des échantillons de lait cru examinés. 


\section{LAITS PASTEURISES}

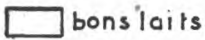

$\%$ rance

曲曲冊 oxdé

oxy dé rance

oxydé betterave.

$x$ rance betherave

betterave ensilage

ensilage pourri

VIIIT
herre bo champign.

gout de vache

$\%$

$\because \because$ gout de betrerave

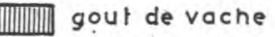

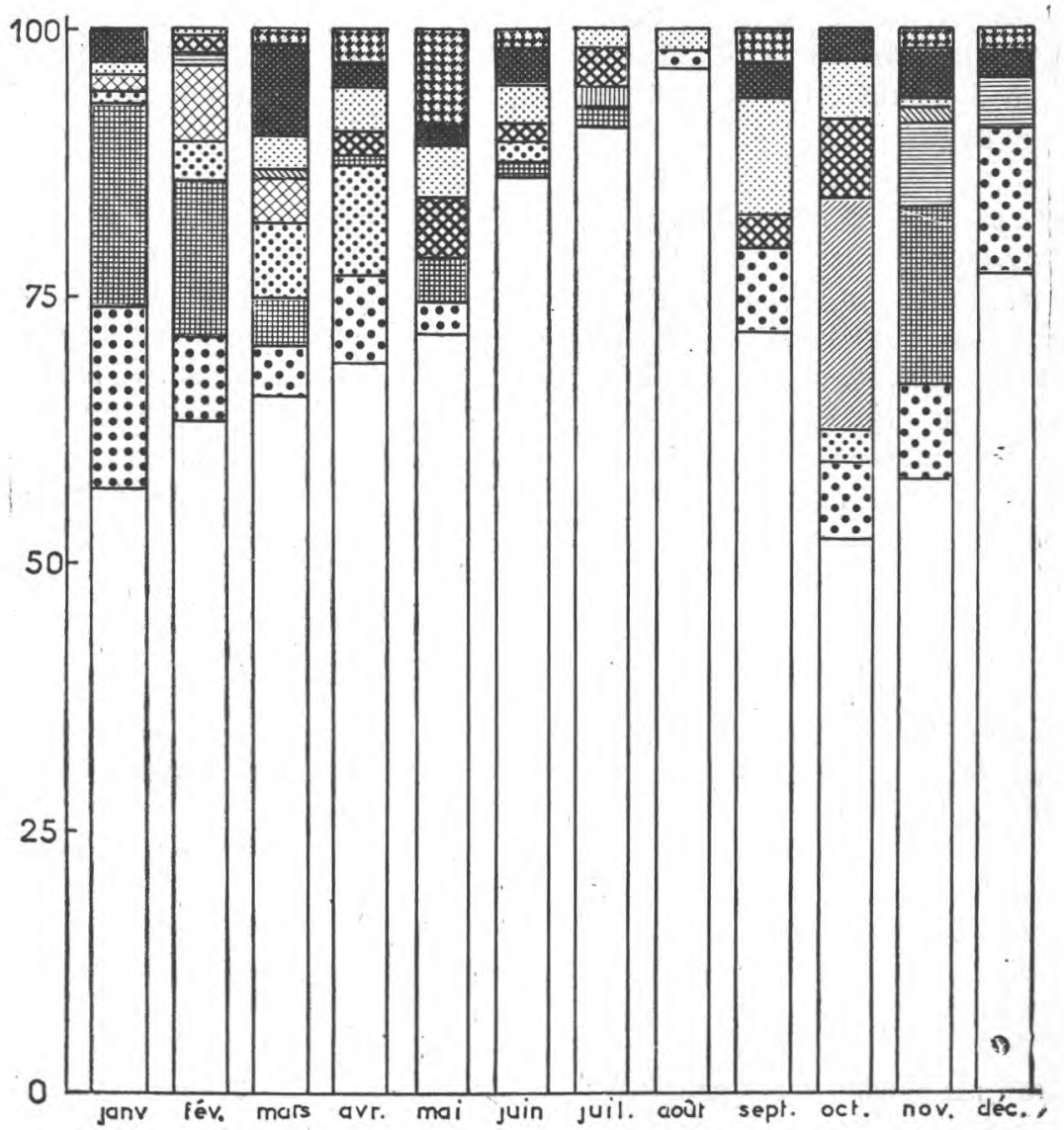

Fig. 1 b. - Proportions de "bons laits » et de laits présentant un défaut de goût, exprimées en pourcentage des échantillons delait pasteurisé examinés. 
des échantillons examinés en mars et sur 13,7 p. 100 des échantillons examinés en avril, n'apparaît plus, en 1956, que sur 11,7 p. 100 des échantillons examinés en février et sur 1 p. 100 des échantillons examinés en avril. Le défaut est absent en mars 1956 et également absent en février et mars 1957.

Une différence existe également entre deux années consécutives en ce qui concerne l'apparition de la saveur oxydée du lait. Ce défaut est relevé pendant les 3 mois de janvier, février et mars sur 7,4 p. 100 des échantillons examinés en 1955, sur 20,5 p.100 des échantillons examinés en 1956 et sur 7,3 p. 100 des échantillons examinés en 1957. En novembre 1955, la saveur oxydée est notée sur 25,3 p. 100 des échantillons examinés, mais en novembre 1956, elle n'est relevée que sur 1 seul échantillon parmi 82 échantillons examinés.

$\mathrm{Au}$ cours de la deuxième période d'examens organoleptiques s'étendant de février 1956 à mars 1957, on a noté un taux plus élevé d'échantillons de lait "mauvais» $(4,3$ p. 100) ou présentant un goût spécial $(3,8$ p. 100), que pendant la première période s'étendant de janvier 1955 à février 1956 (laits mauvais : 0,7 p. 100 ; laits présentant un goût spécial : 2,2 p. 100). Les échantillons de lait rentrant dans la catégorie "bons laits " restaient cependant un peu plus nombreux $(65,5$ p. 100 du total des échantillons examinés pendant la deuxième période que pendant la première période (61 p. 100). Ceci tient en particulier au fait qu'au cours de la deuxième période, les laits présentant une saveur oxydée ou un goût de betterave étaient beaucoup moins nombreux qu'au cours de la première période. (Première période, saveur oxydée 8,3 p. 100, goût de betterave 8,4 p. 100 ; deuxième période, saveur oxydée 3,7 p. 100 , goût de betterave 0,5 p. 100.)

\section{3. - Influence de la pasteurisation}

Nous précisons que la pasteurisation a toujours été effectuée dans les mêmes conditions et toutes précautions ont été prises pour empêcher l'exposition du lait à la lumière solaire.

Dans le tableau I et sur les figures $1 a$ et $1 b$, les résultats concernant les laits crus et les laits pasteurisés étant séparés, l'influence de la pasteurisation est déjà visible, à savoir que la proportion de " bons laits" est plus élevée dans la catégorie des laits pasteurisés que dans celle des laits crus. Le même résultat apparaît dans le tableau II et sur la figure 2 où l'on présente les différents pourcentages d'échantillons de lait examinés (lait cru et lait pasteurisé) rentrant dans la catégorie des "bons laits" ou des laits présentant tel ou tel défaut de goût. Les résultats se rapportant aux deux périodes d'observation sont présentés séparément. 


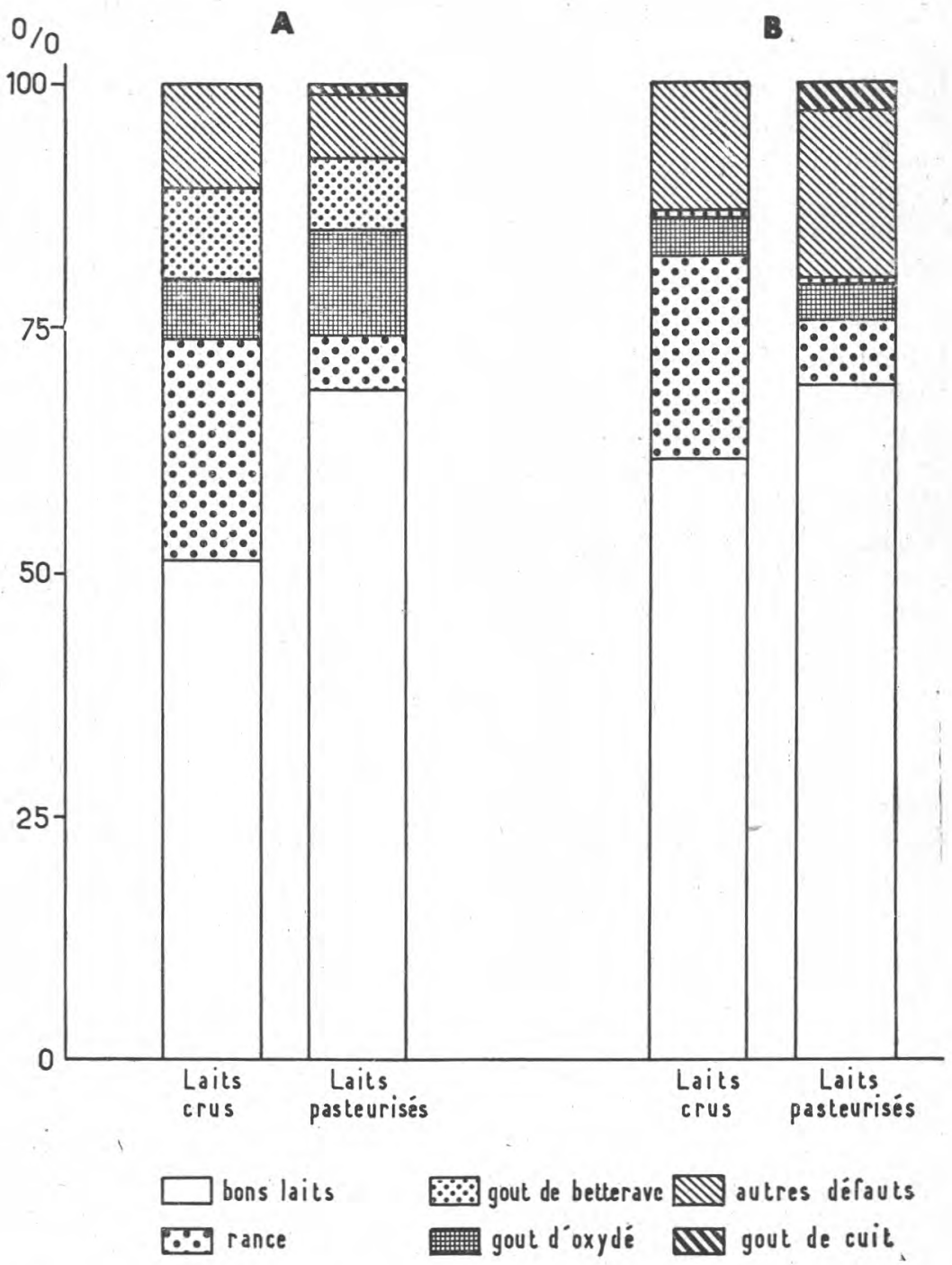

Fig. 2. - Influence de la pasteurisation sur l'apparition des défauts de goût du lait. A : première période d'observation, janvier 1955-janvier 1956. B : deuxième période d'observation, février 1956-mars 1957.

Nous examinerons l'influence de la pasteurisation sur l'apparition des différents défauts de goût dans le lait.

Goût de rance. - Ce défaut apparaît beaucoup plus souvent dans le lait cru que dans le lait pasteurisé. Mais il est cependant présent dans ce dernier. En effet, la pasteurisation des échantillons 
n'avait lieu dans nos essais que 4 à 5 heures après le prélèvement des échantillons de lait de la traite du matin et 16 à 17 heures après le prélèvement des échantillons de lait de la traite du soir. Un début d'hydrolyse de la matière grasse du lait pouvait done se produire pendant ce laps de temps, spécialement dans le cas des laits riches en lipase.

La pasteurisation détruit la lipase du lait et arrête donc tout développement de la lipolyse.

Saveur oxydée. - Ce défaut est plus souvent relevé sur les échantillons de lait pasteurisé que sur les échantillons de lait cru, du moins en ce qui concerne la première période d'observation (janvier 1955-janvier 1956). Nous relevons 10,7 p. 100 d'échantillons de lait pasteurisé présentant une saveur oxydée contre 6,1 p. 100 d'échantillons de lait cru présentant également cette saveur. De février 1956 à mars 1957 (deuxième période d'observation) nous ne retrouvons pas une différence analogue. Il est vrai que pendant cette période, l'apparition de la saveur oxydée a été rarement observée.

Gout de cuit. - Le goût de cuit est particulier au lait pasteurisé. Toutefois nous avons rencontré un échantillon de lait cru présentant un goût de cuit à côté d'un goût de rance (lait de Pivoine, prélèvement du 5 décembre 1956 matin); un goût de caramel a été relevé sur ce même échantillon ayant subi la pasteurisation.

Goût de betterave, goût de nourriture. - Pendant la première période d'observation (janvier 1955-janvier 1956), le goût de betteraves a été relevé un peu plus souvent $(9,1$ p. 100 des échantillons examinés) sur les échantillons de lait cru que sur les échantillons de lait pasteurisé ( 7,6 p. 100 des échantillons examinés). Par contre, pendant la deuxième période d'observation (février 1956mars 1957) le goût de nourriture se rencontre dans 3 p. 100 des échantillons de lait pasteurisé et dans 2 p. 100 des échantillons de lait cru. Mais, d'une façon générale, les goûts divers de nourriture et certains goûts "spéciaux" que nous avons définis par analogie avec des goûts connus, se rencontrent aussi bien dans les échantillons de lait pasteurisé que dans les échantillons de lait eru. Ainsi, au cours d'une même période de quelques semaines (octobre, novembre 1956) nous trouvons, sur des échantillons de lait cru, 4 laits présentant un goût " d'eau de vaisselle ", 8 laits présentant un "goût d'ensilage pourri ", un lait présentant un "goût de foin fermenté ", 2 laits présentant un "goût de vache", 2 laits présentant un "goût d'herbe", et 9 laits présentant un "goût de terre ". Sur les échantillons de lait pasteurisé correspondants, nous relevons 2 laits présentant un "goût d'eau de vaisselle ", 2 laits présentant 
TABLEAU II

Influence de la pasteurisation

PROPORTIONS DES “ BONS LAITS " ET DES LAITS PRÉSENTANT UN DÉFAUT DE GOUT, EXPRIMÉES EN P. GENT DU TOTAL DES ECHANTILLONS EXAMINES

Première période d'observation : janvier 1955 - janvier 1956

\begin{tabular}{|c|c|c|c|c|c|c|c|c|c|c|c|c|c|c|c|}
\hline & Bon & Rance & Oxydé & Better. & $\begin{array}{c}\text { Better. } \\
\text { ensil. }\end{array}$ & $\begin{array}{c}\text { Better. } \\
\text { ensil. } \\
\text { oxydé }\end{array}$ & $\begin{array}{c}\text { Rance } \\
\text { oxydé }\end{array}$ & $\begin{array}{l}\text { Rance- } \\
\text { better. }\end{array}$ & $\begin{array}{l}\text { Oxydé } \\
\text { better. }\end{array}$ & $\begin{array}{c}\text { Goût } \\
\text { de } \\
\text { vache }\end{array}$ & $\begin{array}{c}\text { Goût } \\
\text { d'herbe }\end{array}$ & Spécial & Mauv. & Cuit & Salé \\
\hline Lait eru $\ldots \ldots \ldots \ldots$. & 52,8 & 22,6 & 6,1 & 9,1 & 1,5 & - & 0,4 & 2,4 & 0,8 & 0,2 & 0,4 & 2,6 & 1,1 & - & $I, 3$ \\
\hline Lait pasteurisé $\ldots \ldots \ldots$ & 69,4 & 5,6 & 10,7 & 7,6 & - & 0,5 & - & - & 2,4 & 0,2 & 0,2 & 2,2 & 0,2 & 1,0 & 0,7 \\
\hline
\end{tabular}

Deuxième période d'observation : février 1956 - mars 1957.

\begin{tabular}{|c|c|c|c|c|c|c|c|c|c|c|c|c|c|}
\hline - & Bon & $\begin{array}{c}\text { Plat } \\
\text { fade } \\
\text { passable }\end{array}$ & Rance & Oxydé & Better. & $\begin{array}{l}\text { Better. } \\
\text { oxydé }\end{array}$ & $\begin{array}{l}\text { Rance- } \\
\text { oxydé }\end{array}$ & $\begin{array}{l}\text { Rance- } \\
\text { better. }\end{array}$ & Nourrit. & $\begin{array}{c}\text { Terre, bois, herbe, } \\
\text { eau sale, ensil., } \\
\text { ensil. pourri I }\end{array}$ & $\begin{array}{l}\text { Goût } \\
\text { spécial }\end{array}$ & Mauvais & Cuit \\
\hline Lait cru ...... & 55,9 & 6,0 & 20,4 & 4,0 & 0,6 & - & 0,5 & 0,3 & 2,0 & 3,9 & 3,0 & 3,4 & 0 . \\
\hline Lait pasteurisé & 64,8 & 4,4 & 6,7 & 3,6 & 0,5 & 0,1 & 0,1 & - & 3,0 & 4,4 & 4,5 & 5,3 & 2,6 \\
\hline
\end{tabular}

(1) La saveur salée n'a pas été classée comme un défaut de goût.

(20)


un "goût d'ensilage pourri », 2 laits présentant un "goût d'herbe " et 14 laits présentant un "goût de terre ".

Mauvais laits, goûts spéciaux non définis. - En 1956-1957, on relève davantage de mauvais laits parmi les laits pasteurisés $(5,3$ p. 100) que parmi les laits crus $(3,4$ p. 100). Il en est de même pour les échantillons de lait présentant un goût spécial non défini (4,5 p. 100 des échantillons de lait pasteurisé et 3,0 p. 100 des échantillons de lait cru).

\section{4. - Influence de la Rade}

Les échantillons de lait examinés proviennent de vaches appartenant à trois races: Frisonne Pie Noire (1 079 échantillons, 28 vaches), Normande (880 échantillons, 23 vaches), Pie Rouge de l'Est (212 échantillons, 5 vaches).

Dans le tableau III et sur la figure 3 nous présentons séparément, les résultats des examens organoleptiques effectués sur les échantillons de lait provenant des vaches appartenant à chacune de ces trois races. Pour chaque race, nous avons calculé les différents pourcentages de " bons laits » et de lait présentant un défaut de goût.

On présente séparément les résultats obtenus au cours des deux périodes d'observation et pour la deuxième période (février 1956mars 1957), les résultats se rapportant au lait pasteurisé et au lait cru.

Au cours de la première période d'observation nous constatons que la proportion la plus faible d'échantillons de "bons laits» se rapporte aux échantillons provenant de vaches de race Pie Rouge de l'Est. Ceci est dû à l'apparition fréquente du goût de betterave sur les échantillons provenant de cette race. En effet, pendant la deuxième période d'observation, au cours de laquelle le goût de betterave a été rarement observé (mais cependant toujours plus fréquemment sur les échantillons correspondant à la race Pie Rouge de l'Est) la proportion des "bons laits " est plus élevée parmi les échantillons correspondant à cette race que parmi ceux correspondant aux races Normande ou Frisonne Pie Noire. C'est pour cette dernière race que la proportion de "bons laits", est alors la plus faible parmi les laits crus comme parmi les laits pasteurisés.

Goût de rance. - Nous rencontrons peu de "goût de rance " parmi les échantillons provenant de vaches de race Pie Rouge de l'Est.

Pendant la première période (janvier 1955-janvier 1956) nous notons ce défaut sur 5,6 p. 100 des échantillons provenant des vaches de cette race, alors que nous le relevons sur $15,5 \mathrm{p} .100$ et 


\section{TABLEAU III}

Influence de la race des vaches laitières

\section{PROPORTIONS DES "BONS LAITS " ET DES LAITS PRESENTANT UN DEFAUT DE GOUT EXPRIMEES, POUR GHAQUE RACE}

DE VAGHES LAITIËRES EN P. GENT DU TOTAL DES ECHANTILLONS EXAMINÉS GORRESPONDANT A GHACUNE DES RACES

Première période d'observation : janvier 1955 - janvier 1956 .

\begin{tabular}{|c|c|c|c|c|c|c|c|c|c|c|c|c|c|c|c|}
\hline Race & Bon & Rance & Oxydé & Better. & $\begin{array}{c}\text { Better. } \\
\text { ensil. }\end{array}$ & $\begin{array}{l}\text { Better. } \\
\text { ensil. } \\
\text { oxydé }\end{array}$ & $\begin{array}{l}\text { Rance- } \\
\text { oxydé }\end{array}$ & $\begin{array}{l}\text { Rance- } \\
\text { better. }\end{array}$ & $\begin{array}{l}\text { Oxydé- } \\
\text { better. }\end{array}$ & $\begin{array}{c}\text { Goût } \\
\text { d'herbe }\end{array}$ & $\begin{array}{l}\text { Goût } \\
\text { de } \\
\text { vache }\end{array}$ & $\begin{array}{c}\text { Goût } \\
\text { spécial }\end{array}$ & $\begin{array}{l}\text { Mau- } \\
\text { vais }\end{array}$ & Cuit & Salé \\
\hline Normande $\ldots \ldots \ldots \ldots$ & 66,9 & 17,1 & 6,0 & 5,8 & - & - & - & 1,1 & 0,5 & 0,3 & - & 1,3 & 0,5 & 0,5 & $I, 8$ \\
\hline Frisonne Pie Noire ..... & 63,6 & 15,5 & 10,0 & 5,1 & 0,6 & - & 0,6 & 1,1 & 0,9 & 0,6 & 0,3 & 1,4 & - & 0,3 & - \\
\hline Pie Rouge de l'Est .... & 36,7 & 5,6 & 9,8 & 22,7 & 3,5 & 1,4 & - & 2,1 & 6,3 & - & 0,7 & 7,7 & 2,8 & 0,7 & $I, 4$ \\
\hline
\end{tabular}

Deuxième période d'observation : février 1956 - mars 1957.

\begin{tabular}{|c|c|c|c|c|c|c|c|c|c|c|c|c|c|c|c|c|c|c|c|c|}
\hline Race & 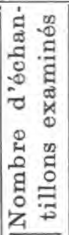 & Bon & 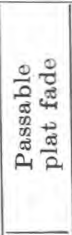 & 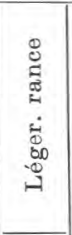 & 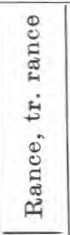 & 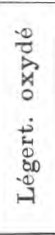 & 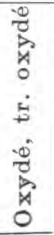 & 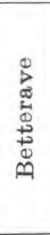 & 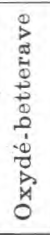 & 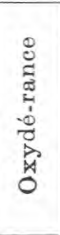 & 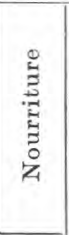 & 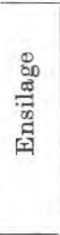 & 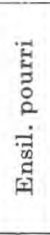 & 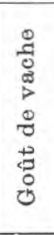 & 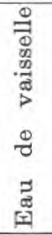 & 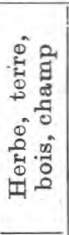 & 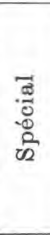 & 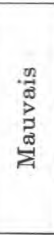 & Cuit & Salé \\
\hline $\begin{array}{l}\text { Lait cru: } \\
\quad \text { Normande ....... }\end{array}$ & & & & & & & & & & & & & & & & & & & & \\
\hline $\begin{array}{l}\text { Normande } \\
\text { Frisonne Pie Noire }\end{array}$ & $\begin{array}{l}251 \\
365\end{array}$ & $\begin{array}{l}63,3 \\
49,0\end{array}$ & $\begin{array}{l}6,0 \\
6,4\end{array}$ & $\begin{array}{c}6,4 \\
10,0\end{array}$ & $\begin{array}{r}9,5 \\
14,8\end{array}$ & $\begin{array}{l}1,6 \\
1,4\end{array}$ & $\begin{array}{l}2,0 \\
2,6\end{array}$ & 二 & E & $\begin{array}{l}0,8 \\
0,3\end{array}$ & $\begin{array}{l}2,0 \\
3,1\end{array}$ & $\overline{0,5}$ & $\overline{1,1}$ & $\begin{array}{l}0,8 \\
0,8\end{array}$ & $\begin{array}{l}0,4 \\
0,8\end{array}$ & $\begin{array}{l}0,8 \\
2,4\end{array}$ & $\begin{array}{l}3,2 \\
2,4\end{array}$ & $\begin{array}{l}3,2 \\
4,4\end{array}$ & - & $\begin{array}{l}I, 6 \\
4, T\end{array}$ \\
\hline Pie Rouge de l'Est. & 35 & 75,9 & 3,0 & 3,0 & - & 3,0 & - & 6,1 & - & - & 3,0 & - & - & - & - & 3,0 & 3,0 & - & - & - \\
\hline Lait pasteurisé : & & & & & & & & & & & & & & & & & & & & \\
\hline Normande ...... & 243 & 69,2 & 5,4 & 2,0 & 2,6 & 0,8 & 2,0 & 0,4 & - & - & 2,0 & - & 0,4 & - & 0,8 & 1,6 & 3,3 & 8,7 & $0,8-8,2$ & 3,3 \\
\hline Frisonne Pie Noire & 358 & 61,9 & 3,9 & 2,7 & 4,4 & 0,6 & 2,8 & 0,3 & 0,3 & 0,3 & 3,4 & 0,6 & 1,4 & 一 & - & 2,8 & 4,5 & 6,7 & $3,4-9,5$ & $5, I$ \\
\hline Pie Rouge de l'Est. & 35 & 62,8 & 2,9 & - & - & - & 2,9 & 5,7 & - & - & 11,3 & - & - & - & 一 & 2,9 & 8,6 & 2,9 & $-5,8$ & $I_{7}, 0$ \\
\hline
\end{tabular}

Les échantillons correspondant aux proportions portées en italique ont été classés dans la catégorie " bons laits » (cf. texte, 2e partie, A2-examen organoleptique) 


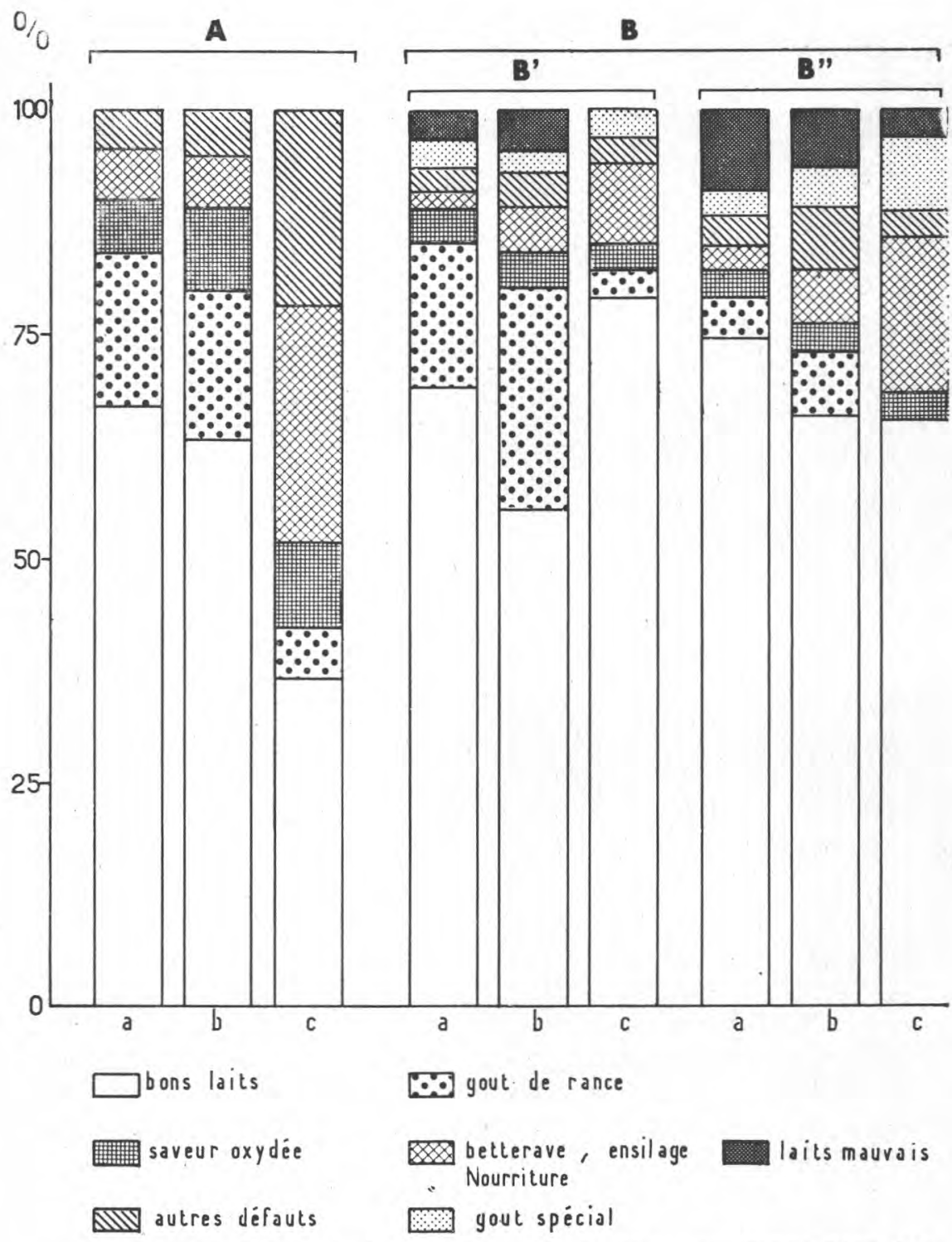

Fig. 3. - Influence de la race des vaches laitières sur l'apparition des défauts de goût du lait. A : première période d'observation, janvier 1955 janvier 1956. B : deuxième période d'observation, février 1956-mars 1957. B' : laits crus. B' : laits pasteurisés. $a$ : race Normande ; $b$ : race Frisonne Pie Noire; $c$ : race Pie Rouge de l'Est.

17,1 p. 100 des échantillons provenant des vaches de race Frisonne Pie Noire et de race Normande. 
Pendant la deuxième période (février 1956-mars 1957), nous ne relevons le "goût de rance" que sur 3 p. 100 des échantillons de lait cru examinés, provenant des vaches de race Pie Rouge de l'Est. Aucun échantillon de lait pasteurisé provenant de vaches de cette race ne présente le goût de rance.

Si l'on étudie les résultats se rapportant aux échantillons provenant des vaches de race Normande et Frisonne Pie Noire on constate que de janvier 1955 à janvier 1956, le goût de rance a été un peu plus souvent observé dans les échantillons de lait provenant des vaches de race Normande $(17,1$ p. 100) que dans cèux provenant des vaches de race Frisonne Pie Noire (15,5 p. 100). Par contre, de février 1956 à mars 1957, c'est l'inverse que l'on constate : 24,8 p. 100 des échantillons de lait cru provenant de vaches de race Frisonne Pie Noire et 15,9 p. 100 des échantillons provenant de vaches de race Normande, ont présenté le goût de rance. Le lait d'un petit nombre de vaches ayant été examiné au cours de la deuxième période, il est permis de penser que l'influence de la race a été masquée par l'influence, peut-être plus importante, de l'individu.

Saveur oxydée. - De février 1956 à mars 1957, nous avons observé beaucoup moins souvent la saveur oxydée que de janvier 1955 à janvier 1956. Cependant nous trouvons au cours de ces deux périodes d'observation, que le pourcentage d'échantillons présentant ce défaut est plus élevé parmi les échantillons provenant des vaches de race Frisonne Pie Noire que parmi ceux provenant des vaches des autres races.

Goût de nourriture. - De janvier 1955 à janvier 1956, un goût d'ensilage a été noté, mêlé au goût de betteraves, dans la proportion de 3,5 p. 100, 0,6 p. 100 et 0 p. 100 des échantillons examinés et provenant respectivement des vaches de race Frisonne Pie Noire, Pie Rouge de l'Est et Normande.

De février 1956 à mars 1957 , le goût de nourriture a été plus fréquemment observé sur les échantillons provenant de vaches de race Frisonne Pie Noire et de race Pie Rouge de l'Est que sur ceux provenant de vaches de race Normande; ainsi, on a trouvé respectivement, pour les races Frisonne Pie Noire, Pie Rouge de l'Est et Normande, 3,1 p. 100, 3 p. 100 et 2 p. 100 des échantillons de lait examinés présentant le goût de nourriture. Ces pourcentages se sont élevés à 3,4 p. $100,11,4$ p. 100 et 2 p. 100 respectivement pour les laits pasteurisés.

Les défauts que nous avons appelés "goût d'ensilage " et "goût d'ensilage pourri » ont été relevés sur des échantillons de lait cru provenant de vaches Frisonne Pie Noire seules et sur les 8 cas 
signalés de ces mêmes défauts concernant les laits pasteurisés, 7 ont été relevés sur des échantillons de lait provenant de vaches de race Frisonne Pie Noire.

Les défauts de goût que l'on a groupés sous la rubrique " herbe, terre, feuilles mortes, bois" se rencontrent moins fréquemment parmi les échantillons de lait provenant de vaches appartenant à la race Normande, que parmi les échantillons de lait provenant de vaches appartenant aux deux autres races Frisonne Pie Noire et Pie Rouge de l'Est.

\section{5. - Qualité comparée du lait du soir ET DU LAIT DU MATIN}

En 1955-1956, on a prélevé régulièrement des échantillons de lait du soir et de lait du matin provenant de chacune des vaches examinées.

Dans le tableau IV, on a porté l'ensemble des résultats concernant les laits crus et pasteurisés. On constate que la proportion de "bons laits" est inférieure pour les laits prélevés le soir ; ceux-ci sont plus fréquemment rances. Ce résultat peut être attribué à une réelle moins bonne qualité des laits du soir, ou tenir au fait que les laits du soir peuvent s'altérer, plus précisément rancir, dans le laps de temps qui précède la pasteurisation.

On a done comparé d'une part, les laits crus du matin et du soir et, d'autre part, les laits pasteurisés du matin et du soir. Les laits crus comme les laits pasteurisés provenant de la traite du soir, restent à la glacière 12 heures de plus que les laits correspondant du matin, puisqu'ils subissent ensemble l'examen organoleptique.

En ce qui concerne les laits crus, on trouve une plus grande proportion des "bons laits" le matin $(57,2$ p. 100) que le soir $(46,4$ p. 100). Cet écart est expliqué, pour la plus grande part, par la proportion plus élevée d'échantillons de laits rances $(27,0$ p. 100) dans les laits du soir que dans ceux du matin (20,3 p. 100) et pour une part plus faible par les défauts de goûts spéciaux un peu plus nombreux le soir que le matin. Par contre, la saveur oxydée se rencontre un peu plus souvent le matin que le soir.

En ce qui concerne les laits pasteurisés, la proportion de bons laits est équivalente soir et matin : 68,6 p. 100 des échantillons prélevés le soir et 70,2 p. 100 des échantillons prélevés le matin. Cependant, et nous en avons déjà expliqué la raison, nous rencontrons davantage de laits présentant le défaut de rance le soir que le matin : 7,8 p. 100 contre 3,5 p. 100 . Par contre, le défaut d'oxydé apparaît nettement plus souvent le matin $(13,4$ p, 100) que le soir (7,8 p. 100). 


\section{TABLEAU] IV}

PROPORTIONS DES « BONS LAITS » ET DES LAITS PRÉSENTANT UN DÉFAUT DE GOUT, DANS LE LAIT DE LA TRAITE DU MATIN ET LE LAIT DE LA TRAITE DU SOIR. (PROPORTIONS EXPRIMÉES EN P. GENT DU TOTAL DES ECHANTILLONS EXAMINÉS)

Première période d'observation : janvier 1955 - janvier 1956.

\begin{tabular}{|c|c|c|c|c|c|c|c|c|c|c|c|c|c|c|c|}
\hline & $\begin{array}{l}\text { Bon } \\
\text { plat } \\
\text { fade }\end{array}$ & Rance & Oxydé & Better. & $\begin{array}{l}\text { Better. } \\
\text { ensil. }\end{array}$ & $\begin{array}{l}\text { Better. } \\
\text { ensil. } \\
\text { oxydé }\end{array}$ & $\begin{array}{l}\text { Rance } \\
\text { better. }\end{array}$ & $\begin{array}{c}\text { Goût } \\
\text { de } \\
\text { vache }\end{array}$ & $\begin{array}{c}\text { Goût } \\
\text { d'herbe }\end{array}$ & $\begin{array}{l}\text { Rance } \\
\text { oxydé }\end{array}$ & $\begin{array}{l}\text { Oxydé } \\
\text { better. }\end{array}$ & Spécial & $\begin{array}{l}\text { Mau- } \\
\text { vais }\end{array}$ & Cuit & Salé (1) \\
\hline Lait du matin .. & 64,5 & 11,0 & 9,5 & 8,3 & 0,7 & 0,2 & 1,2 & - & - & 0,2 & 1,7 & 2,0 & 0,2 & 0,5 & $I, 2$ \\
\hline Lait du soir ... & 56,5 & 18,5 & 6,3 & 9,5 & 1,0 & 0,2 & 1,2 & 0,5 & 0,7 & 0,2 & 1,7 & 2,7 & 0,7 & 0,3 & $I, 0$ \\
\hline
\end{tabular}

Deuxième période d'observation : février 1956 - mars 1957.

\begin{tabular}{|c|c|c|c|c|c|c|c|c|c|c|c|c|c|c|c|}
\hline , & $\begin{array}{l}\text { Bon } \\
\text { plat } \\
\text { fade }\end{array}$ & Rance & Oxydé & Better. & $\begin{array}{c}\text { Better. } \\
\text { ensil. }\end{array}$ & $\begin{array}{l}\text { Better. } \\
\text { ensil. } \\
\text { oxydé }\end{array}$ & $\begin{array}{l}\text { Rance } \\
\text { better. }\end{array}$ & $\begin{array}{l}\text { Rance } \\
\text { better. }\end{array}$ & $\begin{array}{l}\text { Oxydé } \\
\text { better. }\end{array}$ & $\begin{array}{l}\text { Goût } \\
\text { de } \\
\text { vache }\end{array}$ & $\begin{array}{c}\text { Goût } \\
\text { d'herbe }\end{array}$ & $\begin{array}{c}\text { Goût } \\
\text { spécial }\end{array}$ & $\begin{array}{l}\text { Mau- } \\
\text { vais }\end{array}$ & Cuit & Salé (1) \\
\hline \multirow{3}{*}{$\begin{array}{l}\text { Lait cru: } \\
\quad \text { lait du matin. } \\
\quad \text { Lait du soir }\end{array}$} & & & & & & 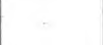 & & & & & & & & & \\
\hline & 57,2 & 20,3 & 5,7 & 9,6 & 1,4 & & 2,4 & 0,5 & 1,0 & - & - & 1,4 & 0,5 & - & 0,9 \\
\hline & 46,4 & 27,0 & 4,8 & 10,6 & 1,9 & & 2,4 & 0,5 & 1,0 & 0,5 & 0,5 & 3,4 & 1,0 & - & $I, 9$ \\
\hline \multirow{3}{*}{$\begin{array}{l}\text { Lait pasteurisé : } \\
\text { Lait du matin } \\
\text { Lait du soir . }\end{array}$} & & & & & & & & & & & & & & & \\
\hline & 70,2 & 3,5 & 13,4 & 6,9 & - & 0,5 & - & - & 2,0 & - & - & 2,5 & - & 1,0 & $I, 5$ \\
\hline & 68,6 & 7,8 & 7,8 & 7,9 & - & 0,5 & - & - & 2,9 & 0,5 & 1,0 & 2,0 & 0,5 & 0,5 & - \\
\hline
\end{tabular}

(1) Les échantillons de lait " salé » ont été classés dans la catégorie " bon, plat, fade ». 
Les autres défauts de goût que présentent les laits pasteurisés apparaissent avec la même fréquence soir et matin.

\section{6. - Influence du stade de lactation}

Pendant la période janvier 1955-janvier 1956, les examens organoleptiques ont porté sur des échantillons de laits provenant de toutes les vaches en lactation, mais on n'a pas examiné régulièrement, comme cela a été fait en 1956-1957, le lait de plusieurs vaches au cours d'une lactation complète.

Cependant en 1955, on connaît pour chaque échantillon le nombre de jours qui sépare le jour du vêlage du jour du prélèvement. Si ce nombre moyen de jours se rapportant à des échantillons présentant un certain défaut de goût est très différent de la moyenne générale de ce même délai, on peut admettre qu'il existe une relation entre ce défaut de goût et le stade de lactation.

La moyenne générale qui sépare le jour du vêlage du jour du prélèvement pour l'ensemble des échantillons est de 145 jours. Elle est de 164 jours pour les échantillons présentant le goût de rance, de 115 jours pour ceux présentant le goût de betterave, et seulement de 60 jours pour les échantillons présentant une saveur oxydée.

Au cours de la deuxième période d'observation nous avons examiné régulièrement le lait de 6 vaches (Pervenche $21 \mathrm{H}$, Cabale $6 \mathrm{~N}$, Hortensia $22 \mathrm{H}$, Pivoine $8 \mathrm{H}$, Pietje $23 \mathrm{H}$, Unité $26 \mathrm{H}$ ) du début à la fin de la lactation, le lait de Laura $32 \mathrm{H}$, au cours d'une lactation à l'exception des deux premiers mois, le lait de Baby $101 \mathrm{~N}$ au cours des dix derniers mois, et des cinq premiers mois de la lactation suivante et le lait de Belladone $30 \mathrm{H}$ au cours des sept derniers mois d'une lactation et des trois premiers mois de la lactation suivante. Les laits de Pastille $9 \mathrm{~N}$ et d'Eglantine $18 \mathrm{H}$ ont été observés régulièrement depuis les vêlages de février et mars 1956 , mais les lactations n'étaient pas terminées en mars 1957 lorsque nous avons arrêté les examens.

Nous portons dans le tableau $V$ et sur la figure 4 les résultats des examens organoleptiques des échantillons correspondant aux deux premiers et aux deux derniers mois de lactation. Il est facile de constater que le lait est moins bon pendant les deux derniers mois de la lactation ("bons laits», 39,2 p. 100) que pendant les deux premiers mois ("bons laits " 71,8 p. 100). Le défaut de rance est très souvent noté en fin de lactation. Les derniers échantillons sont parfois franchement mauvais. Par contre, certains défauts de goût autres que le goût de rance se rencontrent davantage dans le lait produit pendant les deux premiers mois de la lactation. 
REGHERGHES GOMPARATIVES SUR LA GROISSANGE PONDÉRALE ET L'ÉTAT DU SQUELETTE DE JEUNES RATS AYANT REGU DU LAIT AGTINISÉ OU DU MEME LAIT N'AYANT PAS ETÉ SOUMIS A GE TRAITEMENT

Planche No 4

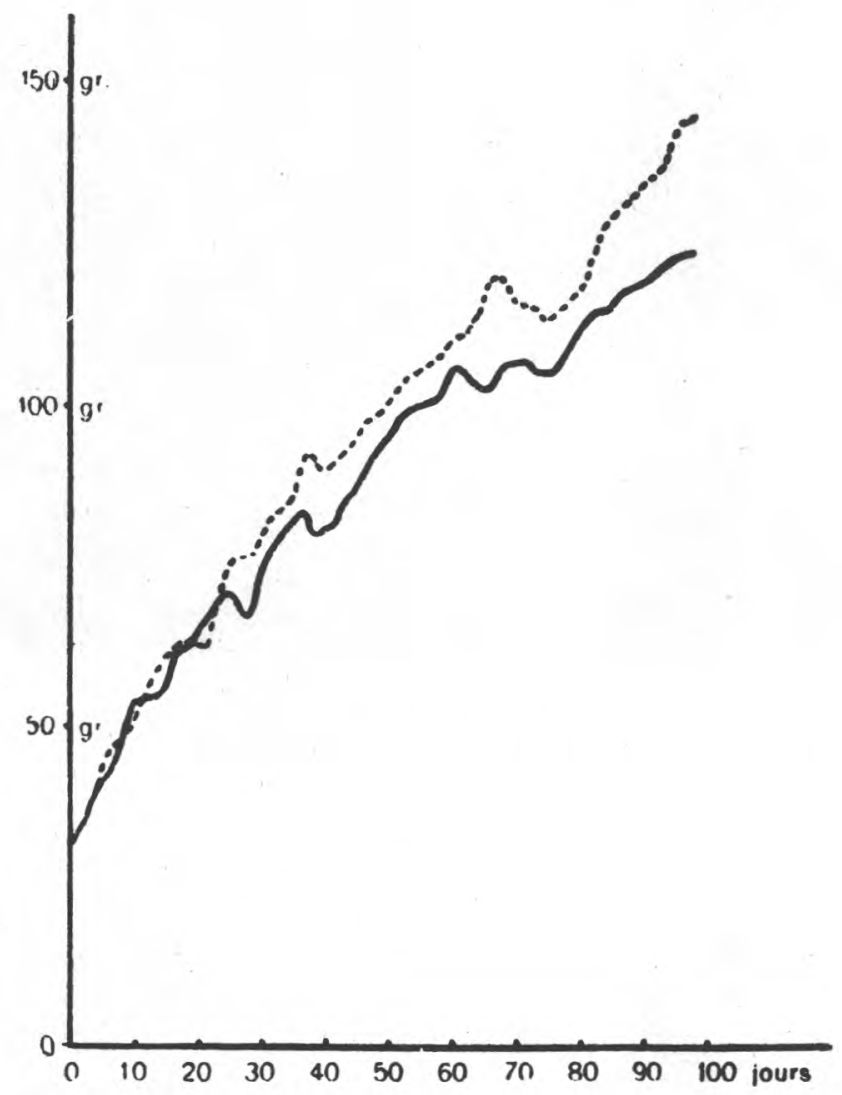

Essais préventifs sur lait actinisé

15 gr pain +2 cc lait actinisé $\{=$ trait pointillé Essais préventifs sur lait non actinisé

15 gr pain +2 cc lait non actinisé $=$ trait plein

Ces expériences mettent en évidence l'amélioration de la valeur nutritive du lait ACTINISE par rapport au lait cru.

Des résultats semblables sont obtenus sur le sérum ou le lait écrémé destiné à l'alimentation des animaux.

Produit « Actintse $》 \rightarrow$ Produit c en Bomme Samé 》

Constructeur en France : 
IADLALU V PROPORTIONS DES "BONS LAITS " ET DES LAITS PRÉSENTANT UN DEFAUT DE GOUT OU UNE SAVEUR SALEE, PENDANT LES
DEUX PREMIERS ET LES DEUX DERNIERS MOIS DE LAGTATION (LAIT CRU)

Pendant les deux premiers mois de lactation

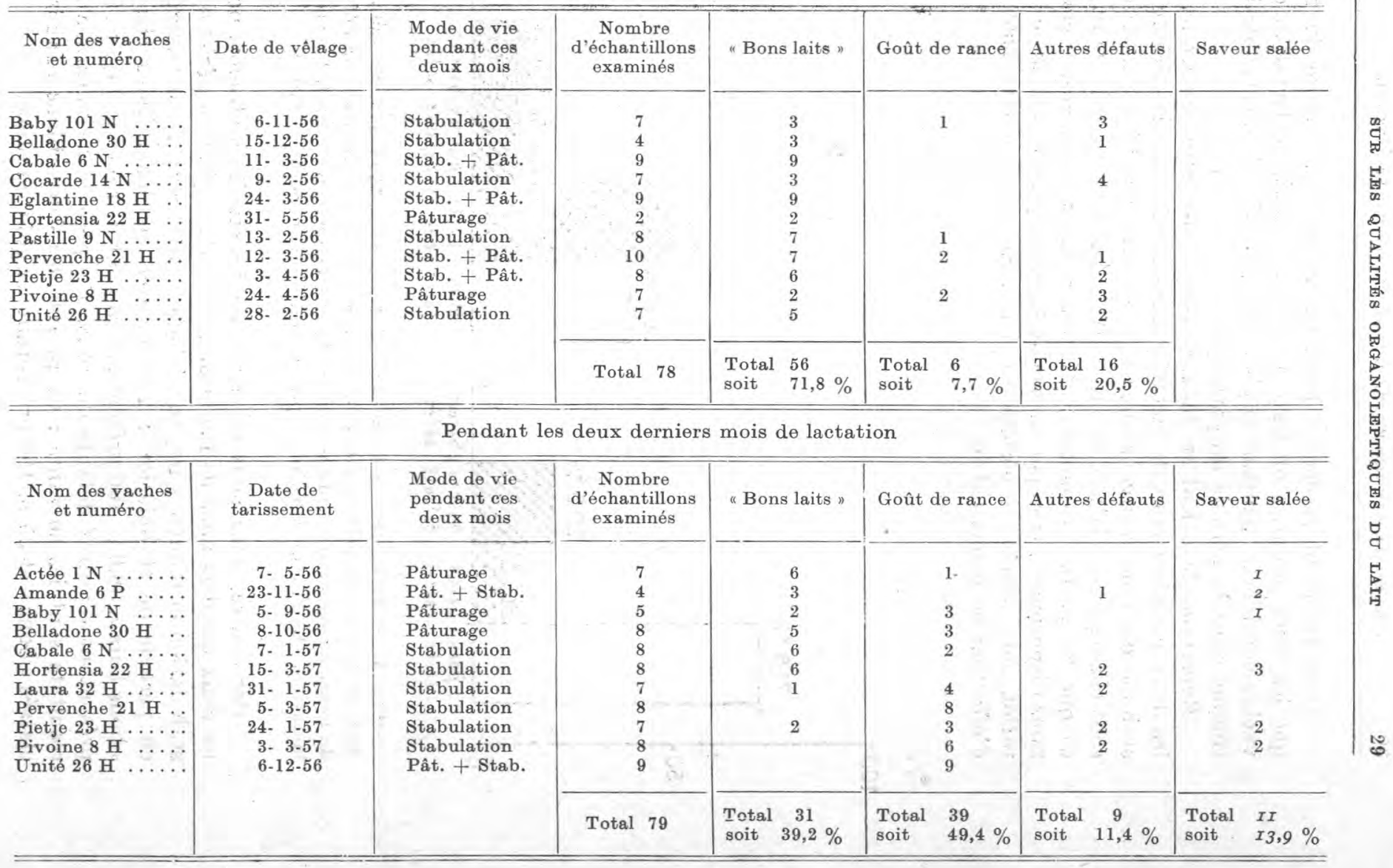


Dans le calcul précédent nous ne tenons pas compte du fait que les deux premiers ou les deux derniers mois de lactation des vaches considérées peuvent se placer dans la période de stabulation comme dans la période de pâturage.

Reprenons le calcul en tenant compte de cette remarque. Si les deux premiers mois de lactation sont situés dans la période de stabulation, nous trouvons que 64 p. 100 des échantillons examinés sont classés "bons laits", que 6 p. 100 présentent un goût de rance et que 30 p. 100 présentent d'autres défauts. Malheureusement, nous disposons de trop peu de cas où les deux premiers mois de lactation sont situés en période de pâturage pour nous permettre d'effectuer ce même calcul.
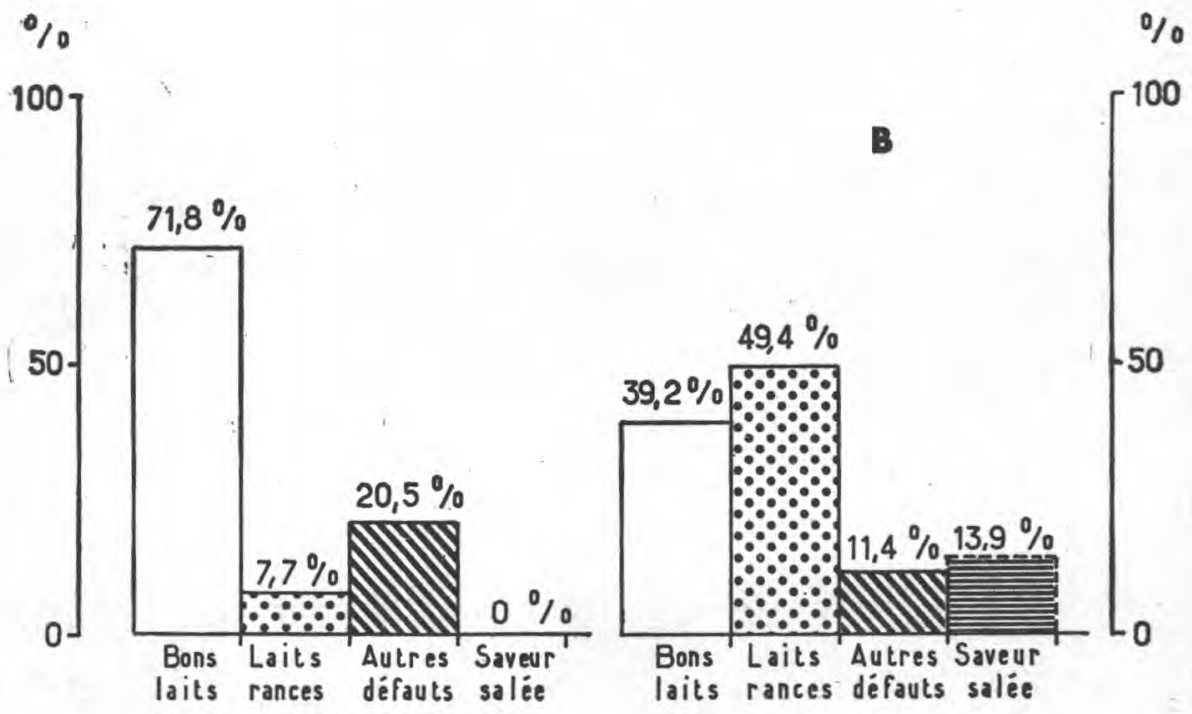

Fig. 4. - Influence du stade de lactation sur l'apparition des défauts de goût du lait. Proportions de « bons laits» et de laits présentant un défaut de goût, au cours des deux premiers (A) et des deux derniers (B) mois de lactation. (La saveur salée n'est pas considérée comme un défaut.)

Par contre, nous pouvons comparer les résultats obtenus pour les deux derniers mois de lactation situés d'une part en période de stabulation et d'autre part, en période de pâturage. Nous trouvons en période de stabulation : 27,2 p. 100 des échantillons classés " bons laits ", 58,0 p. 100 présentant un goût de rance et 14,8 p. 100 présentant d'autres défauts de goût. En période de pâturage les résultats sont les suivants : 65 p. 100 des échantillons examinés classés " bons 》 et 35 p. 100 présentant un goût de rance. 
Nous confirmons donc les résultats portés dans le tableau IV.

Il existe une nette différence d'une vache à une autre en ce qui concerne l'apparition des défauts de goût en fin de lactation. Cette différence existe aussi, si l'on considère la période entière de lactation.

\section{TABLEAU VI}

Première période d'observation : janvier 1955-janvier 1956

CGARTS ENTRE LES PROPORTIONS DES " BONS LAITS ", DE LAITS PRÉSENTANT UN GOUT DE RANCE, DE BETTERAVE OU UNE SAVEUR OXYDÉE, GALGULEES SUR L'ENSEMBLE DES FCHANTILLONS PROVENANT D'UNE SEULE VACHE ET GES MEMES PORPORTIONS GALGULEES SUR L'ENSEMBLE DES EGHANTILLONS PROVENANT DE TOUTES LES AUTRES VACHES. ON NE RETIENT POUR LA COMPARAISON QUE LES EGHANTILLONS PRÉLEVES LES MEMES JOURS

\begin{tabular}{|c|c|c|c|c|c|}
\hline $\begin{array}{c}\text { Race et nom } \\
\text { des vaches }\end{array}$ & $\begin{array}{c}\text { «Bons laits" } \\
\text { en } \%\end{array}$ & $\begin{array}{c}\text { Goût de rance } \\
\text { en } \%\end{array}$ & $\begin{array}{l}\text { Goût de bet- } \\
\text { terave en } \%\end{array}$ & $\begin{array}{r}\text { Saveur } \\
\text { en }\end{array}$ & $\begin{array}{l}\text { oxydée } \\
\%\end{array}$ \\
\hline \multicolumn{6}{|l|}{ Normande: } \\
\hline Actée ........ & $+5,1$ & $+7,5$ & $-11,3$ & - & 4,7 \\
\hline Babou ........ & $+0,4$ & $+2,4$ & $+\quad 1,9$ & - & 4,0 \\
\hline Baby $101 \ldots \ldots$ & $-8,7$ & $+31,7$ & $-10,3$ & - & 0,7 \\
\hline Bandol $116 \ldots$. & $+19,9$ & $-0,1$ & $-7,6$ & - & 4,1 \\
\hline Cabale ...... & $-9,5$ & $-7,3$ & $+16,7$ & - & 0,2 \\
\hline Caline ....... & $+15,2$ & $-0,8$ & & - & 2,7 \\
\hline Cigale ........ & $+15,1$ & $+4,6$ & $-8,2$ & - & 5,8 \\
\hline Cocarde $\ldots \ldots$. & $+20,5$ & $+\quad 1,9$ & $-10,7$ & - & 3,1 \\
\hline Dolly $\ldots \ldots \ldots$ & $+3,0$ & $+6,1$ & $-1,5$ & - & 6,1 , \\
\hline Joliette $\ldots \ldots \ldots$ & $-6,4$ & $+14,9$ & $-1,0$ & - & 5,7 \\
\hline Linotte ....... & $+8,8$ & $+1,5$ & $-7,9$ & - & 1,1 \\
\hline Pastille ...... & $+22,2$ & $-12,6$ & $-1,6$ & 一 & 0,8 \\
\hline \multicolumn{6}{|l|}{ Frisonne Pie Noire: } \\
\hline Borgia ......... & $+\quad 4,5$ & $-9,5$ & $-1,5$ & +1 & 10,8 \\
\hline Belladone..... & $+11,0$ & $+\quad 1,9$ & $-5,6$ & 一 & 5,1 \\
\hline Begonia ...... & $+7,4$ & $-6,1$ & $+8,0$ & - & 1,1 \\
\hline Betty $\ldots \ldots \ldots$ & $+\quad 3,9$ & $+6,1$ & $-8,2$ & - & 1,1 \\
\hline Biscara ....... & $-15,5$ & $+15,4$ & $-0,3$ & + & 9,4 \\
\hline Eglantine ..... & $+25,2$ & $+\quad 0,2$ & $-3,6$ & -1 & 10,1 \\
\hline Hortensia ..... & $+24,3$ & $+\quad 0,9$ & $-\quad 2,9$ & + & 1,5 \\
\hline Laura ....... & - 7,2 & $+\quad 0,6$ & $-6,9$ & +1 & 13,1 \\
\hline Libellule ...... & $+18,7$ & $+\quad 0,5$ & $-13,4$ & + & 1,7 \\
\hline Pivoine $\ldots . .$. & $+14,5$ & $+4,6$ & $-\quad 1,9$ & - & 5,9 \\
\hline Pietje ....... & $-21,0$ & $+2,6$ & $-\quad 1,6$ & + & 7,7 \\
\hline Unité $\ldots \ldots \ldots$ & $+18,5$ & $-10,1$ & $-0,3$ & - & 0,9 \\
\hline \multicolumn{6}{|l|}{ Pie Rouge de l'Est: } \\
\hline Amande $\ldots .$. & $-17,6$ & $-13,6$ & $+8,6$ & + & 7,2 \\
\hline Aïda $\ldots \ldots \ldots \ldots$ & $-22,5$ & $-10,9$ & $+11,3$ & + & 6,8 \\
\hline
\end{tabular}




\section{7. - Influence de l'individu}

a) Première période d'observation : janvier 1955 à janvier 1956

Pendant la première période d'observation, nous avons rapproché les examens portant d'une part, sur les échantillons de lait provenant d'une vache déterminée et portant d'autre part, sur l'ensemble des échantillons de lait provenant des autres vaches; tous ces échantillons étant prélevés les mêmes jours. Ces résultats sont exprimés en pourcentage de la totalité des échantillons examinés de part et d'autre, et sont portés dans le tableau VI.

Le pourcentage de "bons laits" pour les vaches suivantes est nettement plus élevé que le pourcentage de "bons laits " relevé pour l'ensemble des autres vaches :

- Pastille $9 \mathrm{~N}$, Cocarde $14 \mathrm{~N}$, Bandol $116 \mathrm{~N}$, de race Normande.

- Eglantine $18 \mathrm{H}$, Hortensia $22 \mathrm{H}$, Libellule, Unité $26 \mathrm{H}$ de race Frisonne Pie Noire.

Le pourcentage de "bons laits» est nettement inférieur au pourcentage relevé pour l'ensemble du troupeau, pour les vaches suivantes :

- Baby $101 \mathrm{~N}$, Joliette $15 \mathrm{~N}$, de race Normande.

- Biscara $29 \mathrm{H}$, Pietje $23 \mathrm{H}$, Laura $32 \mathrm{H}$ de race Frisonne Pie Noire.

- Amande 6 P, Aida 5 P, de race Pie Rouge de l'Est.

Les laits de Baby $101 \mathrm{~N}$ et Joliette $15 \mathrm{~N}$, de race Normande, et celui de Biscara $29 \mathrm{H}$, de race Frisonne Pie Noire, présentent beaucoup plus souvent que l'ensemble des échantillons, un goût de rance.

Les laits de Borgia $28 \mathrm{H}$, Biscara $29 \mathrm{H}$, Laura $32 \mathrm{H}$, Pietje $23 \mathrm{H}$, de race Frisonne Pie Noire et ceux d'Amande $6 \mathrm{P}$ et d'Aida 5 P de race Pie Rouge de l'Est ont plus souvent que l'ensemble des échantillons, une saveur oxydée; on ne relève cette particularité sur le lait d'aucune vache de race Normande.

Le lait produit par Amande $6 \mathrm{P}$ et Aïda $5 \mathrm{P}$ présente plus souvent que l'ensemble des échantillons examinés, un goût de betterave. On relève le même fait pour le lait de Bégonia $20 \mathrm{H}$ (Frisonne Pie Noire) et de Cabale $6 \mathrm{~N}$ (Normande).

\section{b) Deuxième période d'observation: février 1956-mars 1957}

Pendant la deuxième période d'observation, la saveur oxydée et le goût de betterave ont été beaucoup moins souvent relevés que pendant la première période d'observation. Il est à remarquer que lorsqu'on a relevé le défaut de goût de betterave, il s'est agi dans 
cette deuxième période, 4 fois sur 7, du lait d'Amande ou d'Aïda, de race Pie Rouge de l'Est. On a pu cependant comparer au cours de cette deuxième période, les proportions des "bons laits " et de laits présentant le goût de rance, d'une part, pour les échantillons se rapportant à une vache examinée régulièrement, et d'autre part, pour l'ensemble des échantillons examinés pendant la même période. On obtient le tableau VII.

On constate que les proportions les plus élevées de "bons laits " se rencontrent pour les vaches suivantes : Pastille $9 \mathrm{~N}$ (race Normande), Hortensia $22 \mathrm{H}$, Belladone $30 \mathrm{H}$ (race Frisonne Pie Noire). Les proportions les plus faibles pour les vaches suivantes : Cocarde $14 \mathrm{~N}$, Baby $101 \mathrm{~N}$ (race Normande), Pervenche $21 \mathrm{H}$ (race Frisonne Pie Noire).

\section{TABLEAU VII}

Deuxième période d'observation : février 1956-mars 1957

PROPORTIONS DES EGHANTILLONS DE " BONS LAITS n .ET DES EGHANTILLONS DE LAIT PRESENTANT UN GOUT DE RANGE, GALGULEES D'UNE PART, SUR L'ENSEMBLE DES EGHANTILLONS PROVENANT D'UNE SEULE VACHE ET PRELEVES PENDANT UNE GERTAINE PÉRIODE ET GALGULEES D'AUTRE PART, SUR L'ENSEMBLE DES ÉCHANTILLONS DE LAIT PROVENANT DE TOUTES LES VAGHES ET EXAMINÉS PENDANT LA MEME PÉRIODE (TÉMOIN)

\begin{tabular}{|c|c|c|c|c|c|c|c|c|}
\hline \multirow{3}{*}{$\begin{array}{c}\text { Nom } \\
\text { des vaches }\end{array}$} & \multicolumn{4}{|c|}{ Bons laits } & \multicolumn{4}{|c|}{ Laits présentant un goût de rance } \\
\hline & \multicolumn{2}{|c|}{ Lait cru } & \multicolumn{2}{|c|}{ Lait pasteurisé } & \multicolumn{2}{|c|}{ Lait cru } & \multicolumn{2}{|c|}{ Lait pasteurisé } \\
\hline & $\begin{array}{c}\text { Indivi- } \\
\text { duel } \\
\text { en } \%\end{array}$ & $\begin{array}{l}\text { Témoin } \\
\text { en \% }\end{array}$ & $\begin{array}{c}\text { Indivi- } \\
\text { duel } \\
\text { en } \%\end{array}$ & $\begin{array}{c}\text { Témoin } \\
\text { en } \%\end{array}$ & $\begin{array}{c}\text { Indivi- } \\
\text { duel } \\
\text { en } \%\end{array}$ & $\left\{\begin{array}{c}\text { Témoin } \\
\text { en } \%\end{array}\right.$ & $\begin{array}{c}\text { Indivi- } \\
\text { duel } \\
\text { on } \%\end{array}$ & $\begin{array}{c}\text { Témoin } \\
\text { en } \%\end{array}$ \\
\hline Amande $6 \mathrm{P}$ & 79,3 & 71,0 & 65,5 & 70,0 & 0 & 13,7 & 0 & 3,6 \\
\hline Baby $101 \mathrm{~N} \ldots$ & 40,0 & 62,7 & 71,8 & 71,8 & 47,5 & 22,2 & 10,3 & 6,6 \\
\hline Belladone $30 \mathrm{H}$ & 87,5 & 69,2 & 71,9 & 74,9 & 9,4 & 15,5 & 3,1 & 5.2 \\
\hline Cabale $6 \mathrm{~N} \ldots$ & 86,5 & 64,1 & 73,0 & 67,9 & 8,1 & 19,2 & 0 & 6,0 \\
\hline Cocarde $14 \mathrm{~N}$. & 51,5 & 68,2 & 57,5 & 68,0 & 30,3 & 16,1 & 15,1 & 4,5 \\
\hline Eglantine $18 \mathrm{H}$ & 66,6 & 62,0 & 66,6 & 69,7 & 26,6 & 21,7 & 11,1 & 7.7 \\
\hline Hortensia $22 \mathrm{H}$ & 72,7 & 57,4 & 81,2 & 68,8 & 0 & 26,4 & 0 & 10,1 \\
\hline Laura $32 \mathrm{H} \ldots$ & 51,2 & 62,9 & 64,3 & 68,0 & 18,6 & 20,9 & 4,7 & 5,8 \\
\hline Pastille $9 \mathrm{~N} \ldots$ & 91,8 & 60,5 & 87,2 & 69,1 & 8,2 & 21,5 & 0 & 6,8 \\
\hline Pervenc. $21 \mathrm{H}$ & 28,8 & 62,1 & 62,8 & 68,1 & 60,0 & 21,7 & 15,4 & 7,4 \\
\hline Pietje $23 \mathrm{H}$. & 60,0 & 63,2 & 76,5 & 68,9 & 17,1 & 20,1 & 0 & 6,4 \\
\hline Pivoine $8 \mathrm{H}$. & 47,3 & 61,0 & 52,6 & 67,1 & 36,8 & 22,8 & 18,4 & 9,1 \\
\hline Unité $26 \mathrm{H} \ldots$ & 51,4 & 65,9 & 54,3 & 68,9 & 31,4 & 17,7 & 14,3 & 5,7 \\
\hline
\end{tabular}


Les laits provenant d'Unité $26 \mathrm{H}$, de Pervenche $21 \mathrm{H}$, de Pivoine $8 \mathrm{H}$, de Cocarde $14 \mathrm{~N}$ et de Baby $101 \mathrm{~N}$, présentent plus souvent que l'ensemble des échantillons, un goût de rance. Par contre le lait produit par Amande $6 \mathrm{P}$ et Hortensia $22 \mathrm{~N}$ ne présente jamais, en 1956-1957, ce même défaut.

Le lait de Cocarde $14 \mathrm{~N}$ était considéré en 1955-1956, comme excellent; il ne possédait plus cette qualité en 1956-1957. L'état de santé de Cocarde a été mauvais dès juillet 1956.

Les différences que l'on observe entre les résultats se rapportant au lait d'une vache et ceux se rapportant à l'ensemble des échantillons aussi bien pour les pourcentages de bons laits, que pour la fréquence d'apparition des défauts de goût, sont toujours plus accentuées pour les laits crus que pour les laits pasteurisés.

\section{8. - Influence De LA NOURRIture}

Depuis la rentrée à l'étable jusqu'à la mise à l'herbe, la nourriture distribuée aux vaches laitières n'est pas uniforme. Elle est à base de foin, de betteraves, d'ensilage et de concentré (ce dernier étant distribué proportionnellement à la quantité de lait produit par chaque vache), mais les rations des trois premiers éléments varient au cours de l'hiver et varient également d'une vache à l'autre, par suite des expériences d'alimentation poursuivies par la Station de Recherches sur l'Elevage.

D'autre part, pendant la période de pâturage, il arrive fréquemment que des aliments d'appoint soient distribués aux vaches laitières (pulpe de betteraves, fanes de carottes, choux, herbe coupée, luzerne en vert) lorsque l'herbe des prairies s'avère insuffisante.

Il est donc difficile d'établir une relation précise entre la nourriture distribuée aux vaches laitières et l'apparition des défauts de goût dans le lait. Seule une étude comportant des examens organoleptiques fréquents (journaliers par exemple) effectués sur le lait produit par différentes vaches ayant vêlé à des dates voisines, et dont l'alimentation est exactement connue, peut permettre d'obtenir des résultats valables.

Notre étude nous permet cependant de remarquer que la répercussion de l'alimentation sur la qualité organoleptique du lait varie en fonction de l'individualité des vaches laitières. Ainsi, le lait de Pastille 9 N, vache appartenant à la race Normande, semble particulièrement résistant à l'apparition des défauts de goût : malgré une consommation journalière réelle d'ensilage voisine de 15 à 20 kilos, tous les échantillons de lait examinés pendant la 
période correspondante se classent dans la catégorie des "bons laits» à l'exception d'un seul échantillon de lait présentant un "goût de rance".

\section{9. - INFluence de L'État de santé de la Vache laitik̀re}

Nous avons noté, au cours de nos observations, un goût de rance accentué et souvent un "mauvais goût » sur des échantillons de lait provenant de vaches dont l'état de santé n'était pas satisfaisant (Cocarde, Vinette, Pigeonne).

Nous avons noté à de nombreuses reprises, que le lait provenant de vaches atteintes de mammite, présentait une saveur salée.

Nous n'avons pas constaté qu'un traitement aux antibiotiques effectué en cas de mammite entraîne l'apparition de défaut de goût particulier dans le lait.

\section{DISGUSSION ET GONGLUSION}

Au cours de l'expérimentation conduite à Jouy-en-Josas, nous avons retrouvé les défauts de goût signalés dans le lait par les auteurs étrangers.

Nous noterons cependant que la saveur oxydée se rencontre moins fréquemment sur le lait pasteurisé en France, que dans les pays anglo-saxons. Ceci peut être lié au fait que la pasteurisation industrielle s'effectue chez nous, très souvent, à une température voisine de $90^{\circ} \mathrm{C}$. Par contre, le goût de cuit est un défaut à peu près général mais auquel le consommateur français est habitué, puisque l'ébullition du lait reste une pratique courante dans de nombreux foyers.

Il nous a été possible, au cours de cette étude, de confirmer l'influence d'un certain nombre de facteurs déjà signalés par les auteurs étrangers. Mais il est certain que l'influence propre, exercée par l'alimentation, le stade de lactation ou la race des vaches laitières est difficile à établir d'après les informations recueillies au cours de notre étude. En particulier, nous ne pouvons pas conclure en ce qui concerne l'influence de l'alimentation, sur l'apparition des défauts de goût dans le lait et il sera nécessaire que cette question fasse l'objet d'études ultérieures.

En ce qui concerne l'influence du stade de lactation, nous pensons que la conclusion que nous donnons est valable : à savoir que le goût de rance est un défaut caractéristique de la fin de lactation et que ce défaut est plus accentué lorsque les deux derniers mois de lactation se situent en période de stabulation. Nous ne pensons cependant pas que le fait, pour les vaches laitières, de 
rester à l'étable, ou le fait de recevoir une alimentation d'hiver, suffisent à provoquer l'apparition du goût de rance, puisque lors des deux premiers mois de lactation même quand ils se situent en période de stabulation, on ne relève que rarement le goût de rance sur les échantillons de lait examinés.

Nous avons relevé que la saveur oxydée semble apparaître davantage sur les échantillons de lait provenant de vaches en début de lactation. Nous avons noté aussi que le lait produit le matin était plus sensible à l'oxydation que le lait du soir. Peut-on en conclure qu'une relation existe entre la quantité de lait produit et la susceptibilité de ce lait au développement de la saveur oxydée?

Nous avons, d'autre part, noté au cours de notre étude, que le lait produit par les vaches de race Normande présentait moins souvent que le lait produit par les vaches appartenant aux deux autres races (Frisonne Pie Noire et Pie Rouge de l'Est) le défaut de "saveur oxydée". Ceci provient-il d'une plus faible production laitière des vaches de race Normande, qu'il faudrait démontrer, ou doit-on envisager l'action de substances dites "protectrices» vis-à-vis du développement de la saveur oxydée ? Nous savons en effet que le lait produit par les vaches de race Normande est toujours plus coloré que le lait produit par les vaches de race Frisonne Pie Noire ou Pie Rouge de l'Est, du moins en période de stabulation, seule période pendant laquelle le lait présente une "saveur oxydée ". Cette question de l'influence de la coloration de la matière grasse du lait sur la susceptibilité de ce dernier à l'oxydation a été plusieurs fois évoquée par les auteurs. Il est possible que l'adjonction de carotène à la ration alimentaire d'hiver des vaches laitières soit à retenir.

Produire un lait dont la qualité organoleptique soit sans cesse améliorée, doit rester le souci primordial de l'éleveur. Pour obtenir ce résultat, les plus grands soins doivent être apportés à la surveillance sanitaire des vaches laitières, à leur "confort", au choix de leur alimentation. Il se peut cependant que malgré ces précautions, la qualité organoleptique de certains laits reste mauvaise. L'éleveur devrait alors éviter l'incorporation de tels laits au lait destiné à la consommation.

Nous remercions la Station de Recherches sur l'Elevage, qui a mis $\grave{a}$ notre disposition les renseignements de tous ordres concernant l'ensemble du troupeau examiné.

Nous remercions égatement MM. Fauconneau et Dupin, qui ont bien voulu nous prêter leur concours lors des examens organoleptiques. 


\section{SUMMARY}

In the first section a review of the extensive literature on flavour defects in milk is given.

In the second section, the authors give the results of their own observations on milk from individual cows in the experimental herd of the C.N.R.Z. Jouy-en-Josas, during the years 1955, 1956 and beginning of 1957 .

Oxidized, rancid and feed flavours were the predominant flavour defects. The influence of various factors including season, diet, stage of lactation and breed of cattle on the tendency of milk to develop these faulty flavours was studied.

\section{RÉFÉRENCES BIBLIOGRAPHIQUES}

[1] G. Mocquot. Aspects techniques et hygiéniques du problème des laits destinés à la consommation. Ann. Nut. Al., 1955, 9, no 4 .

[2] B. L. Herrington. The control of rancidity in milk. J. Dairy Sci., 1956, 39, 1613 .

[3] W. C. Brown et L. M. Thurston. A review of oxidation in milk and milk products as related to flavor. J. Dairy Sci. 1940, 23, 629 .

[4] G. R. Gremnank. The oxidized flavor in milk and dairy products : a review. J. Dairy Sci., 1948, 31, 913.

[5] C. I. Kruisheer. Defects in flavor of liquid milk caused by oxidative processes. Neth. Milk. Dairy J., 1952, 6, 242.

[6] C. H. LEA. Oxidation defects of milk and dairy products. XIII ${ }^{\mathrm{e}}$ Congrès International de Laiterie. La Haye, 1953, vol. III. Sect. 4, 1037.

[7] R. R. Riel et H. H. Sommer. Oxidized flavor in milk. I. Review literature. Institut d'Oka, éditeur. La Trappe. P. Q., Canada, 1954.

[8] J. W. STULL. The effect of light on activated flavor development and on the constituents of milk and its products : a review. J. Dairy Sci., 1953, 36, 1153.

[9] S. Patton. Browning and associated changes in milk and its products : a review. J. Dairy Sci., 1955, 38, 457.

[10] C. T. Roland, C. M. Sorensen et R. Whitaker. A study of oxidized flavor in commercial pasteurized milk. J. Dairy Sci., 1937, 20, 213.

[11] C. L. ROADHOUSE et J. L. HeNDERson. Flavors of milk and their control. University of California. Bulletin 595, 1935.

[12] O. AUle et T. Storgards. The resistance of milk to the development of oxidized flavour, as influenced by season. XIIIe Congrès international de Laiterie. La Haye, 1953. Sect. IV, sujet 2, p. 1076.

[13] W. H. Chilson, W. H. Martin et D. B. Parrish. The relationship of ascorbic acid to the development of oxidized flavor in market milk. J. Dairy Sci., 1949, 32, 306. 
[14] G. M. Trout et C. G. Jessing. Ascorbic acid and oxidized flavor in milk. I. Distribution of ascorbic acid and occurence of oxidized flavor in commercial graded A raw, in pasteurized irradiated and in pasteurized milk throughout the year. J. Dairy Sci., 1939, 22, 271.

[15] J. A. Nelson et G. M. Trout. Judging and grading milk. Olsen Publis. Co Milwaukee 12. Wis., 1951.

[16] W. KRUkowsky et J. K. LoosLr. Further studies on the influence of tocopherol supplementation on the vitamin content of the milk fat, stability of milk and fat production. J. Dairy Sci., 1952, 35, 834 .

[17] B. R. WEINstein et G. M. Trout. Susceptibility of milk from various dairy breeds to homogenization induced rancidity. Milch. Agric. Exp. Sta. Quart. Bull., 1950, 32, 311.

[18] H. Hvidstenh, J. V. Mehlum et Simonsen. Influence of feed on oxidized flavour development in milk. I. Feeding cod liver oil and herring meal. Meieriposten 41, 9, 145-151; 10, 170-173; 11, 186-190. II. Effects of feeding large quantities of hay and silage and the effect of pasture. Meieriposten 41, 14, 241-245;15, 265263, 1952 (ou D.S.A., 1952, p. 554).

[19] B. R. Weinstein et G. M. Trout. The solar activated flavor of homogenized milk. I. Susceptibility of individual cow's milk. J. Dairy Sci., 1951, 34, 554.

[20] W. L. Dunkley. Hydrolytic rancidity in milk. I. Surface tension and fat acidity as measures of rancidity. J. Dairy Sci., 1951, 34, 515 .

[21] H. Fresdeen, J. E. Bowstead, W. L. Dunkiey et L. M. Smith. Hydrolytic rancidity in milk. II. Some managements and environmental factors influencing lipolysis. J. Dairy Sci, , 1951, 34, 521.

[22] W. R. Thomas, W. J. Harper et I. A. Gould. Lipase activity in fresh milk as related to portions of milk drawn and fat globule size. J. Dairy Sci., 1955, 38, 315.

[23] W. C. Brown, L. M. Thurston et R. B. Dustman. Oxidized flavor in milk. IV. Studies of the relation of the feed of the cow to oxidized flavor. J. Dairy Sci., 1937, 20, 133.

[24] Sternitz et H. H. Sommer. The oxidation of butterfat. II. The composition of the fat in the relation to its susceptibility toward oxidation. J. Dairy Sci., 1937, 20, 265.

[25] F. KaGi. Undesirable odours and flavours in milk. Mitt. Lebensm. Hyg. Bern, 1950, 41, 65.

[26] P. H. Tracy et H. A. RueHe. The relation of certain plant processes to flavor development in market milk. J. Dairy Sci., 1931, 14, 250.

[27] R. Honore et H. Hvidsten. Influence of feed on oxidized flavor development in milk. III. Addition of copper to the feed. Meieriposten, 1952, 41 (17), 309-313.

[28] W. G. Epple et B. E. Horall. The relation of copper and ascorbic acid to oxidized flavor in market milk. J. Dairy Sci., 1943, 26, 525. 
[29] A. De Vlegechauwer, H. Hendrick et E. Waliez. Contribution à l'étude sur le goût d'oxydation du lait. XIIIe Congrès International de laiterie, La Haye, 1953, Sect. IV, sujet 2, p. 1072.

[30] L. M. Thurston, W. C. Brown et R. B. Dustman. Oxidized flavor in milk. II. The effects of homogenization, agitation and freezing of milk on its subsequent susceptibility to oxidized flavour development. J. Dairy Sci., 1936, 19, 671.

[31] V. N. KRUKowskY et P. F. SHARP. Effect of shaking on the lipolysis of cow's milk. J. Dairy Sci., 1938, 21, 671.

[32] B. L. Herrington. Lipase : a review. J. Dairy Sci., 1954, 37, 775.

[33] V. N. KRUkOW sKy et B. L. HerRINGton. Studies on the lipase action. II. The activation of milk lipase by temperature changes. J. Dairy Sci., 1939, 22, 137.

[34] D. H. Nelson et C. D. DaHLe. The relation of iodine and peroxyde numbers to oxidized flavor of milk. J. Dairy Sci., 1940, 23, 391.

[35] C. L. RoAdhouse and G. A. Konsteme. Contribution to the knowledge on the taste of milk. J. Dairy Sci., 1929, 12, 421.

[36 a] S. Patron, D. A. Forss et E. A. Day. Methylsulfide and the flavor of milk. J. Dairy Sci., 1956, 39, 1469.

[36 b] F. V. KosIkowskr. Free volatile fatty acids in homogenized raw milk. Int. Dairy Cong., 3, 1545. Londres, 1959.

[37] G. MaJer. Molkerei Ztng, Hildesh., 1931, 45.

[38] R. G. JENSEN et M. E. Morgan. Detection of I. monoglycerides in the fat from rancid milk. J. Dairy Sci., 1957, 40, 1199.

[39] V. N. KRUkowsky et B. L. Herrington. Studies on lipase action. VI. The effect of lipolysis upon the flavour score of milk. J. Dairy Sei., 1942, 25, 235.

[40] N. P. TARAssuK et F. R. SмItH. Studies on rancid milk. Relation of surface tension of rancid milk to its acid coagulation. J. Dairy Sci., 1939, 22, 415 .

[41] J. Proks et J. GRoн. Le goût défectueux du lait. Le Lait, 1935, 15, 370.

[42] B. L. Herrington et V. N. KRUkowsky. Studies of lipase. I. Lipase action in normal milk. $J$. Dairy Sci., 1939, 22, 127.

[43] C. H. EckLe et R. H. Shaw. The influence of the stage of lactation on the composition and properties of milk. Bureau of Anim. husb., 1913, bull. 155 .

[44] L. Hiynk. et E. G. Hood. Milk lipase and milk flavor. J. Dairy Sci, $1942,25,389$.

[45] P. L. KeLLY. The lipolytic activity of bovine mammary gland tissue. J. Dairy Sci., 1943, 26, 385.

[46] V, N. KRUkowsky et B. L. Herrington. Studies on lipase action. VII. The influence of the rate of cooling upon the subsequent rate of lipolysis in milk stored at low temperature. J. Dairy Sci., 1942, 25, 241.

[47] N. P. TARAssur et JACK. Lipolytic flavors of milk. Milk plant monthly, 1949, 38, 48. 
[48] N. P. TARASSUK et E. N. Frankel. On the mechanism of activation of lipolysis and the stability of lipase systems of normal milk. J. Dairy S'ci., 1955, 38, 438.

[49] D. C. Rohan et H. H. Sommer. Lipolytic activity in milk and eream. J. Dairy Sci., 1940, 23, 831.

[50] I. A. GouLd. Influence of $p$ H, type of fat and pancreatic extract upon hydrolysis in homogenized milk. J. Dairy Sci., 1941, 24, 779.

[51] E. C. V. Matrick et H. D. Kay. A lipase (tributyrinase) of cow's milk. Occurrence, method of estimating and relation to lactation cycle. J. Dairy Res., 1938, 9, 58.

[52] M. H. Peterson, H. J. Johnson et W. V. Price. Determination of milk lipase. J. Dairy Sci., 1943, 26, 233.

[53] T. W. Albrecht et H. O. JAynas. Milk lipase. J. Dairy Sci., 1955, 38, 137 .

[54] P. L. Kelly. Milk lipase activity, a method for its determination and its relation to the estrus cycle. J. Dairy Sci., 1945, 28, 803.

[55] P. L. Kelly. The determination of lipase from milk extracted with acetone and ether. J. Dairy Sci., 1944, 27, 675.

[56] D. P. Schwartz, I. A. Gould et W. J. Harper. The milk lipase system. II. Effect of formaldehyde. J. Dairy Sci., 1956, 39, 1375.

[57] C. T. Roland et H. A. Trebler. The effect of fat content on oxidized flavor in milk and eream. J. Dairy Sci., 1957, 20, 345.

[58] E. G. Pont. Studies on the origin of oxidized flavour in whole milk. J. Dairy Res., 1952, 19, 316.

[59] M. Kemney et F. J. Dran. Studies on oxidized milk fat. J. Dairy Sci., 1951, 34, 713.

[60] U. Holm, G. Wode et K. E. Thоме. Oily flavour in butter and $\alpha, \beta$, unsaturated earbonyl compounds. Medd, Statens Mejeriforsök no 37,1952 .

[61] K: E. Thome et S. Mattsson. Investigations into the oxidation defects in butter. XIII Congrès Intern. Laiterie. La Haye, 1953, 3, Sect. IV, 1056.

[62] D. A. Forss, E. G. Pont et W. Stark. The volatile compounds associated with oxidized flavor in skim milk. J. Dairy Res., 1955, 22, 91.

[63] D. A. Forss, E. G. Pont et. W. Stark. Further observations on the volatile compounds associated with oxidized flavor in skim milk. J. Dairy Res., 1955, 22, 345.

[64] S. KENDE. Untersuchungen über "ölig ", "talgige ", "schmirgelige" Veranderungen der Milch. Milehw. Forsch.; 1932, 13, 111.

[65] C. D. Dahle et L. S. Palmer. The oxidized flavor in milk from the individual cow. Penna Agric. Exp. Sta. Bull., 1937, 347.

[66] W. C. Brown, R. B. Dustman et L. M. Thurston. Oxidized flavor in milk. V. The effect of metal developed oxidized flavor on the iodine number of the milk fat. $J$. Dairy Sci., 1934, 17, 321.

[67] J. L. HENDERson et C. L. RoAdHousE. Factors influencing the initial induction period in the oxidation of milk fat. J. Dairy Sci., $1934,17,321$. 
[68] G. E. Holm et G. R. Gregnbank. Quantitative aspects of the Kreis test. Industr. Engin. Chem., 1923, 15, 1051.

[69] B. E. Horall. A study of the lecithin content of milk and its products. Ind. Agr. Exp. Sta. Bull., 1935, 401.

[70] L. M. Thurston, W. C. Brown et R. B. Dustman. Oxidized flavor in milk. I. The probable relation of lecithin to oxidized flavor. J. Dairy Sci., 1935, 18, 301.

[71] A. M. Swanson et H. H. Sommer. Oxidized flavor in milk. I. Effect of the development of oxidized on the iodine number of the phospholipid fraction of milk. J. Dairy Sci., 1940, 23, 201.

[72] M. Hietaranta. The oxidation reduction potential in butter and the oxidation of butterfat. Suomen Kemistilehti, 19 B, 125, 1946. Chem. Abst., 1947, 41, 5227.

[73] R. R. Riel et H. H. Sommze. Isolation and eharacterization of some oxidation products associated with oxidized flavor. $J$. Dairy Sci., $1955,38,1215$.

[74] G. H. Beck, C. H. Whitnah et W. H. Martin, Relation of vitamin C, lecithin and earotene of milk to the development of oxidized flavor. J. Dairy Sci., 1939, 22, 17.

[75] 1. A. Gould, W. K. Fox et G. M. Trout. Influence of feeds on lecithin content to susceptibility of milk to copper induced oxidized flavor. Food Res., 1940, 5, 131.

[76] E. S. Guthrie. Development of oxidized flavors in unhomogenized milk and homogenized milk. J. Dairy Sci, 1955, 38, 595.

[77] W. C. Brown, L. M. Thurston et R. B. Dustman. Oxidized flavor in milk. IV. Studies of the relation of the feed of the cow to oxidized flavor. J. Dairy Sci, 1937, 20, 133.

[78] W. C. Brown, A. H. Vanlandingham et C. E. Weakley. Oxidized flavor in milk. VII. Studies of the effect of carotene and ascorbic acid in the feed of the cow on the susceptibility of the milk to metal induced oxidized flavor. J. Dairy Sci., 1939, 22, 345 .

[79] P. F. Sharp, G. M. Trout et E. S. Guthrie. Vitamin C, copper and the oxidized flavor of milk. Milk plant Monthly, 1937, 24, 32.

[80] G. H. Beck, C. H. Whitnah et W. H. Martin. Relation of vitamin C, lecithin and carotene of milk to the development of oxidized flavor. J. Dairy Sci., 1939, 22, 17.

[81] C. M. Trout et C. G. Jessing. Ascorbic acid and oxidized flavor in milk. I. Distribution of ascorbic acid and occurrence of oxidized flavor in commercial grade A raw, in pasteurized irradiated, and in pasteurized milk throughout the year. J. Dairy Sci., 1939, 22.

[82] V. N. KRUKowsKY et E. S. GUthrie. Ascorbic acid oxidation a key factor in the inhibition or promotion of the tallowy flavor in milk. J. Dairy Sci., 1945, 28, 565 .

[83] W. H. Chilson, W. H. Martin et D. B. Parrish. The relationship of ascorbic acid to the development, of oxidized flavor in market milk. $J$. Dairy Sci., 1949, 32, 306. 
[84] F. C. Olson et W. C. Brown. Oxidized flavor in milk. XI. Aseorbic acid, glutathion and hydrogen peroxide as mechanisms for the production of oxidized flavor. J. Dairy Sci., 1942, 25, 1027.

[85] J. A. Anderson. Off flavors milk. A problem of animal nutrition. The milk dealer, octobre 1936, 26, 60,62, 64, 66 .

[86] V. N. KRUkowskY et J. K. Loosir. Further studies on the influence of tocopherol supplementation on the vitamin content of the milk fat, stability of milks and milk and fat production. J. Dairy Sci., 1952, 35, 834 .

[87] R. E. Werb et J. L. Hileman. The relation of the oxidation reduction potential of milk to oxidized flavor. J. Dairy Sci., 1937, 20, 47.

[88] W. L. Davies. The action of strong sunlight on milk (certified milk). Certified milk, 1931, 6, 4.

[89] P. H. Tracy et H. A. RumHz. The relation of certain plant processes to flavor development in market milk. J. Dairy Sci., 1931, 14, 250

[90] F. H. McDowall, A. K. R. MeDowell, N. J. Clemon et M. E. Bell. The effect of sunlight on the nutritional properties and flavour of milk in glass bottles. The Dairy Research Institute (N. Z.). Septembre 1948. Publication $n^{\circ} 211$.

[91] B. R. Weinstein et G. M. Trout. The solar activated flavor of homogenized milk. I. Susceptibility of individual cow's milk. J. Dairy Sci., 1951, 34, 554.

[92] B. R. Weinstein et G. M. Trout. II. The role of oxidation and the effectiveness of certain treatments. J. Dairy Sci., 1951, 34, 559.

[93] J. C. FLAKE, H. C. JACKson et K. G. WeCKEL. Studies on the aotivated flavor of milk. Paper presented at the 33rd Ann. Meet. A.J.D.S., 1938.

[94] B. R. Whinstein et G. M. Trout. III. Effect of deaeration surface area of fat globules and relation of the Kreis test. J. Dairy Sci., $1951,34,565$.

[95] B. R. Weinstein, C. W. Duncan et G. M. Trout. IV. Isolation and characterization of a whey constituent capable of producing the solar-activated flavor. J. Dairy Sci., 1951, 34, 570.

[96] E. O. Herreid, B. Ruskin, G. L. Clark et T. B. Parks. Ascorbic acid and riboflavin destruction and flavor development in milk exposed to the sun in Amber, clear, paper and ruby bottles. $J$. Dairy Sci., 1952, 35, 772.

[97] J. T. Hutron et S. Patron. The origin of sylphydryl groups in milk protein and their contribution to cooked flavor. J. Dairy Sci., $1952,35,699$.

[98] S. Patton. The mechanism of sunlight flavor formation in milk with special reference to methionine and riboflavine. J. Dairy Soi., $1954,37,446$.

[99] C. J. JACK son. Note on the sulphydryl compounds of milk. J. Dairy Res., 1936, 7, 29.

[100] D. V. Josephson et F.J. Doan. Observations on cooked flavor in milk : its source and significance. Milk dealer, 1939, 29, 35. 
[101] S. Patton et D. V. Josephon. Observations on the application of the nitroprussite test to heated milk. J. Dairy Sci., 1949, 32, 398.

[102] R. C. Townley et I. A. Gould. A quantitative study of heat labile sulfides of milk. I. A method of determination and the influence of temperature and time. J. Dairy Sci., 1943, 26, 689.

[103] I. A. GouLd. Compounds formed by heat treatment of milk. Milk plant monthly, 1951, 40, 44.

[104] I. A. Jr Gould and H. H. Sommer. Effect of heat on milk with special reference to the cooked flavor. Mich. Agri. Exp. Sta. Tech. Bull., $1939,164$.

[105] A. T. R. Matтick. J. Agr. Sci., 1924, 17, 388.

[106] E. G. PonT. Seasonal variation in the susceptibility of market milk to oxidized flavour. Aust. J. Dairy Techn., 1958, 13, 146.

[107] A. K. R. McDowell et F. H. McDowall. The vitamin A potency of New Zealand butter. J. Dairy Res., 1953, 20, 76.

[108] A. Françors. Recherche sur le comportement biochimique et physiologique de la vitamine A dans la sécrétion lactée. Thèse Frac. Sci., Paris, 1948.

[109] G. Schwarz, J. Ludwig et E. M. Giessler. Eigenschaften und Zusammensetzung von Kuhmilch in Baden Würtemberg. Milchw. Forsch. Kiel, Heft 3, 1957.

[110] E. S. Guthrie et Brueckner. Cornell Agr. Exp. Sta. Bull., 1933, 606, 10.

[111] V. N. Krukowsky, F. Whiting et J. K. Loosli. Tocopherol, carotenoid and vitamin A content of the milk fat and the resistance of milk to the development of oxidized flavors as influenced by breed and season. J. Dairy Sci. 1950, 33, 791.

[112] B. L. Herrington et V. N. Krukowsky. Studies on lipase action. III. Lipase action in the milk of individual cows. J. Dairy Sci., $1939,22,149$.

[113] J. L. Hileman et F. Courtney. Seasonal variations in the lipase content of milk. J. Dairy Sci., 1935, 18, 247.

[114] N. P. Tarassuk et W. M. Regan. A study of the blood carotene in relation to lipolytic activity of milk. J. Dairy Sei., 1943, 26, 987.

[115] H. Freeden, J. E. Bowstehd, W. L. Dunkley et L. M. Sмith. Hydrolytic raneidity in milk. II. Some management and environmental factors influencing lipolysis. J. Dairy Sci., 1951, 34, 521.

[116] R. Rasmussen, N. B. Gugrrant, A. O. Shaw, R. C. Wellk et S. L. BECKDEL. J. Nutrition, 1936, 11, 425.

[117] W. J. Corbett et P. H. Tracy. The incidence of oxidized flavor in the milk individual cows within one herd. J. Dairy Sci., 1943, 26, 1095 .

[118] Bruekner et E. S. Guthrie. Proc. Intern. Assoc. milk dealers. Lab. Sec. 3-16, 1934.

[119] R. L. King et W. L. Dunklex. Relation of natural copper in milk to incidence of spontaneous oxidized flavor. J. Dairy Sci., 1959, 42,420 . 